\title{
On the Domains of Dirichlet Forms on Metric Measure Spaces
}

\author{
Meng Yang
}

\begin{abstract}
We prove that the domain of the local Dirichlet form is strictly contained in the domain of any stable-like non-local Dirichlet form on general metric measure spaces.

प्राप्रा
\end{abstract}

\section{Introduction}

Let us recall the following classical Sobolev spaces.

$$
\begin{aligned}
& H^{1}\left(\mathbb{R}^{d}\right)=\left\{u \in L^{2}\left(\mathbb{R}^{d}\right): \int_{\mathbb{R}^{d}}|\nabla u(x)|^{2} \mathrm{~d} x<+\infty\right\} \\
& H^{\delta}\left(\mathbb{R}^{d}\right)=\left\{u \in L^{2}\left(\mathbb{R}^{d}\right): \int_{\mathbb{R}^{d}} \int_{\mathbb{R}^{d}} \frac{(u(x)-u(y))^{2}}{|x-y|^{d+2 \delta}} \mathrm{d} x \mathrm{~d} y<+\infty\right\} \text { for } \delta \in(0,1) .
\end{aligned}
$$

We have their characterizations using Fourier transform as follows.

$$
H^{\delta}\left(\mathbb{R}^{d}\right)=\left\{u \in L^{2}\left(\mathbb{R}^{d}\right): \int_{\mathbb{R}^{d}}|\mathcal{F}[u](x)|^{2}|x|^{2 \delta} \mathrm{d} x<+\infty\right\} \text { for } \delta \in(0,1],
$$

where $\mathcal{F}: L^{2}\left(\mathbb{R}^{d}\right) \rightarrow L^{2}\left(\mathbb{R}^{d}\right), u \mapsto \mathcal{F}[u]$ is the Fourier transform which is an isometry. It is easy to check that $H^{\delta}\left(\mathbb{R}^{d}\right)$ is monotone decreasing in $\delta \in(0,1]$, that is, $H^{\delta_{1}}\left(\mathbb{R}^{d}\right) \supseteq H^{\delta_{2}}\left(\mathbb{R}^{d}\right)$ for any $\delta_{1}, \delta_{1} \in(0,1]$ with $\delta_{1}<\delta_{2}$, hence $H^{\delta_{0}}\left(\mathbb{R}^{d}\right) \subseteq \cap_{\delta \in\left(0, \delta_{0}\right)} H^{\delta}\left(\mathbb{R}^{d}\right)$ for any $\delta_{0} \in(0,1]$. A natural question is whether this is equal. However, the answer is NO. Denote $\mathcal{F}^{-1}$ : $L^{2}\left(\mathbb{R}^{d}\right) \rightarrow L^{2}\left(\mathbb{R}^{d}\right)$ as the inverse Fourier transform. Let

$$
u=\mathcal{F}^{-1}\left[\left(x \mapsto \frac{1}{|x|^{\frac{d}{2}+\delta_{0}}} 1_{|x|>1}\right)\right]
$$

Then $u \in \cap_{\delta \in\left(0, \delta_{0}\right)} H^{\delta}\left(\mathbb{R}^{d}\right) \backslash H^{\delta_{0}}\left(\mathbb{R}^{d}\right)$ by the above characterizations.

The main purpose of this paper is to consider similar questions on general metric measure spaces where function spaces serve as the domains of Dirichlet forms.

Let $(K, d, \nu)$ be an $\alpha$-regular metric measure space. Consider the following non-local quadratic form

$$
\begin{aligned}
& \mathcal{E}_{\beta}(u, u)=\int_{K} \int_{K} \frac{(u(x)-u(y))^{2}}{d(x, y)^{\alpha+\beta}} \nu(\mathrm{d} x) \nu(\mathrm{d} y) \\
& \mathcal{F}_{\beta}=\left\{u \in L^{2}(K ; \nu): \int_{K} \int_{K} \frac{(u(x)-u(y))^{2}}{d(x, y)^{\alpha+\beta}} \nu(\mathrm{d} x) \nu(\mathrm{d} y)<+\infty\right\},
\end{aligned}
$$

where $\beta \in(0,+\infty)$ is so far arbitrary. Note that $\mathcal{F}_{\beta}$ is monotone decreasing in $\beta$ and $\mathcal{F}_{\beta}$ may be trivial for very large $\beta$. The critical exponent

$$
\beta^{*}:=\sup \left\{\beta \in(0,+\infty):\left(\mathcal{E}_{\beta}, \mathcal{F}_{\beta}\right) \text { is a regular Dirichlet form on } L^{2}(K ; \nu)\right\}
$$

Date: June 12, 2020

MSC2010: Primary 60J35; Secondary 28A80, 31C25

Keywords: Sobolev space, Dirichlet form, Sierpiński gasket, Sierpiński carpet

The author was very grateful to Dr. Eryan Hu for helpful discussions. The author was very grateful to anonymous suggestions to generalize to general metric measure spaces. 
is called the walk dimension of the metric measure space $(K, d, \nu)$. It holds true on many metric measure spaces that $\beta^{*} \in(0,+\infty), \mathcal{F}_{\beta^{*}}$ is trivial but there lives a local regular Dirichlet form $\left(\mathcal{E}_{\text {loc }}, \mathcal{F}_{\text {loc }}\right)$ on $L^{2}(K ; \nu)$ related to the critical exponent $\beta^{*}$. For example, the Euclidean spaces and a large family of fractal spaces including the Sierpiński gasket and the Sierpiński carpet. It is natural to raise the following questions.

Question 1. Is it true that $\mathcal{F}_{\text {loc }} \subseteq \cap_{\beta \in\left(0, \beta^{*}\right)} \mathcal{F}_{\beta}$ ? Is it true that $\mathcal{F}_{\text {loc }}=\cap_{\beta \in\left(0, \beta^{*}\right)} \mathcal{F}_{\beta}$ ?

Question 2. Given $\beta_{0} \in\left(0, \beta^{*}\right)$, is it true that $\mathcal{F}_{\beta_{0}}=\cap_{\beta \in\left(0, \beta_{0}\right)} \mathcal{F}_{\beta}$ ?

On $\mathbb{R}^{d}, \beta^{*}=2, \mathcal{F}_{\text {loc }}=H^{1}\left(\mathbb{R}^{d}\right)$ and $\mathcal{F}_{\beta}=H^{\beta / 2}\left(\mathbb{R}^{d}\right)$ for $\beta \in(0,2)$. The answers to the above two questions were already given.

First, we consider two typical fractal spaces, that is, the Sierpiński gasket and the Sierpiński carpet. On the Sierpiński gasket, $\beta^{*}=\log 5 / \log 2$ and the existence of a local regular Dirichlet form was given by [4, 10]. On the Sierpiński carpet, $\beta^{*} \approx 2.0969$ whose exact value is still unknown and the existence of a local regular Dirichlet form was given by [1, 13. We give the answer to Question 1 as follows.

Proposition 1.1. On the Sierpiński gasket (SG) and the Sierpiński carpet (SC), we have

$$
\mathcal{F}_{\text {loc }} \varsubsetneqq \bigcap_{\beta \in\left(0, \beta^{*}\right)} \mathcal{F}_{\beta} .
$$

Remarks 1.2. (1) "ᄃ" was used implicitly by Grigor'yan and the author [8, 9, 17] to show the denseness of $\mathcal{F}_{\beta}$ in certain function spaces. It is relatively easy to prove "ᄃ $\subseteq$ ".

(2) The key novelty of the above result is "Ғ". We will prove by constructing explicit functions in $\cap_{\beta \in\left(0, \beta^{*}\right)} \mathcal{F}_{\beta} \backslash \mathcal{F}_{\text {loc. }}$. The construction on the $S G$ is much easier than the construction on the $S C$ due to the facts that the $S G$ is a typical finitely ramified fractal space while the $S C$ is a typical infinitely ramified fractal space. Due to the intrinsically analytic difference between the $S G$ and the $S C$, the construction on the $S G$ can not be applied on the SC at all.

(3) The above result can be in fact covered by the following Proposition 1.3. However, we will give the construction of some explicit functions in $\cap_{\beta \in\left(0, \beta^{*}\right)} \mathcal{F}_{\beta} \backslash \mathcal{F}_{\text {loc }}$ in the proof instead of giving only the existence of such functions in the proof of Proposition 1.3 .

Second, we give conditions to give answers to Question 1 and Question 2 as follows.

Proposition 1.3. Let $(K, d, \nu)$ be an $\alpha$-regular metric measure space satisfying the chain condition and that all metric balls are relatively compact. Let $(\mathcal{E}, \mathcal{F})$ be a conservative regular Dirichlet form on $L^{2}(K ; \nu)$ with a heat kernel $p_{t}(x, y)$ satisfying

$$
\frac{C_{1}}{t^{\alpha / \beta_{0}}} \Phi\left(C_{2} \frac{d(x, y)}{t^{1 / \beta_{0}}}\right) \leq p_{t}(x, y) \leq \frac{C_{3}}{t^{\alpha / \beta_{0}}} \Phi\left(C_{4} \frac{d(x, y)}{t^{1 / \beta_{0}}}\right)
$$

for any $x, y \in K$, for any $t \in\left(0, \operatorname{diam}(K)^{\beta_{0}}\right)$, here $\operatorname{diam}(K):=\sup \{d(x, y): x, y \in K\}$ is infinite if $K$ is unbounded and is finite if $K$ is bounded, where $\beta_{0} \in(0,+\infty)$ is some parameter, $C_{1}, C_{2}, C_{3}, C_{4}$ are some positive constants and $\Phi:(0,+\infty) \rightarrow(0,+\infty)$ is some monotone decreasing function. Then

$$
\mathcal{F} \varsubsetneqq \bigcap_{\beta \in\left(0, \beta_{0}\right)} \mathcal{F}_{\beta} .
$$

Remark 1.4. Grigor'yan and Kumagai [7, Theorem 4.1] proved that under the above settings, the following dichotomy holds.

(a) Either $(\mathcal{E}, \mathcal{F})$ on $L^{2}(K ; \nu)$ is local, $\beta_{0} \in[2, \alpha+1]$ and

$$
\Phi(s) \asymp C \exp \left(-c s^{\frac{\beta_{0}}{\beta_{0}-1}}\right) .
$$

(b) $\operatorname{Or}(\mathcal{E}, \mathcal{F})$ on $L^{2}(K ; \nu)$ is non-local, $\beta_{0} \in(0, \alpha+1]$ and

$$
\Phi(s) \asymp(1+s)^{-\left(\alpha+\beta_{0}\right)} .
$$


If $(\mathcal{E}, \mathcal{F})=\left(\mathcal{E}_{\text {loc }}, \mathcal{F}_{\text {loc }}\right)$ and $\beta_{0}=\beta^{*}$, then this is case (a) and the above result gives the answer to Question 1 . If $(\mathcal{E}, \mathcal{F})=\left(\mathcal{E}_{\beta_{0}}, \mathcal{F}_{\beta_{0}}\right)$ and $\beta_{0} \in\left(0, \beta^{*}\right)$, then this is case (b) and the above result gives the answer to Question 2.

Proposition 1.3 is indeed a consequence of the following result.

Theorem 1.5. Let $(K, d, \nu)$ be a metric measure space. Let $(\mathcal{E}, \mathcal{F})$ be a regular Dirichlet form on $L^{2}(K ; \nu)$ that corresponds to a Hunt process $\left\{X_{t}\right\}$. Let $A$ be the generator of $(\mathcal{E}, \mathcal{F})$ on $L^{2}(K ; \nu)$ which is a non-positive definite self-adjoint operator. For any $\delta \in(0,1)$, let $\left\{X_{t}^{(\delta)}\right\}$ be the $\delta$-subordinated Hunt process that corresponds to a regular Dirichlet form $\left(\mathcal{E}^{(\delta)}, \mathcal{F}^{(\delta)}\right)$ on $L^{2}(K ; \nu)$. Then

$$
\mathcal{F} \subseteq \bigcap_{\delta \in(0,1)} \mathcal{F}^{(\delta)},
$$

and "=" if and only if $A$ is bounded.

Remark 1.6. " $\subseteq$ " is also relatively easy to prove. We will prove " $\subsetneq$ " when $A$ is unbounded by giving the existence of some function in $\cap_{\delta \in(0,1)} \mathcal{F}^{(\delta)} \backslash \mathcal{F}$.

This paper is organized as follows. In Section 2, we prove Proposition 1.1 for the SG. In Section 3, we prove Proposition 1.1 for the SC. In Section 4, we prove Theorem 1.5. In Section 5, we prove Proposition 1.3 .

In this paper, we always assume that $(K, d, \nu)$ is a metric measure space, that is, $(K, d)$ is a locally compact separable metric space and $\nu$ is a Radon measure on $K$ with full support. We use $(\cdot, \cdot)$ to denote the inner product in $L^{2}(K ; \nu)$. If $(\mathcal{E}, \mathcal{F})$ is a closed form on $L^{2}(K ; \nu)$, then we always define $\mathcal{E}(u, u)=+\infty$ for any $u \in L^{2}(K ; \nu) \backslash \mathcal{F}$, hence $\mathcal{E}$ is defined on the whole $L^{2}(K ; \nu)$ rather than a dense subspace $\mathcal{F}$.

NOTATION. The letters $c, C$ will always refer to some positive constants and may change at each occurrence. The sign $\asymp$ means that the ratio of the two sides is bounded from above and below by positive constants. The sign $\lesssim(\gtrsim)$ means that the LHS is bounded by positive constant times the RHS from above (below).

\section{Proof of Proposition 1.1 for the SG}

Let $K$ be the SG in $\mathbb{R}^{2}$, that is, let $p_{0}=0, p_{1}=(1,0), p_{2}=\left(\frac{1}{2}, \frac{\sqrt{3}}{2}\right)$ and $f_{i}(x)=\frac{1}{2}\left(x+p_{i}\right)$, $x \in \mathbb{R}^{2}, i=0,1,2$, then $K$ is the unique non-empty compact set in $\mathbb{R}^{2}$ satisfying $K=$ $\cup_{i=0}^{2} f_{i}(K)$, see Figure 1. Let $|\cdot|$ be the Euclidean metric in $\mathbb{R}^{2}$ and $\nu$ the normalized Hausdorff measure of dimension $\alpha=\frac{\log 3}{\log 2}$ on $K$. Then $(K,|\cdot|, \nu)$ is an $\alpha$-regular compact metric measure space.

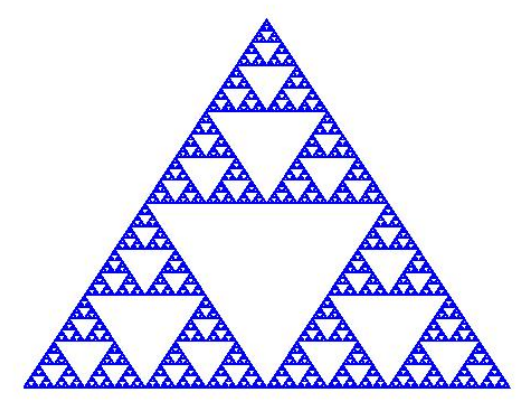

Figure 1: The SG in $\mathbb{R}^{2}$

Denote $l(S)$ as the set of all real-valued functions on a set $S$.

We list the characterization of local regular Dirichlet form on the SG as follows, see [10, 11, 12] for reference.

Let $W_{0}=\{\emptyset\}$ and

$$
W_{n}=\left\{w=w_{1} \ldots w_{n}: w_{i}=0,1,2, i=1, \ldots, n\right\} \text { for any } n \geq 1 .
$$


For any $w^{(1)}=w_{1}^{(1)} \ldots w_{m}^{(1)} \in W_{m}, w^{(2)}=w_{1}^{(2)} \ldots w_{n}^{(2)} \in W_{n}$, let

$$
w^{(1)} w^{(2)}=w_{1}^{(1)} \ldots w_{m}^{(1)} w_{1}^{(2)} \ldots w_{n}^{(2)} \in W_{m+n} .
$$

Let $V_{0}=\left\{p_{0}, p_{1}, p_{2}\right\}$ and $V_{n+1}=\cup_{i=0}^{2} f_{i}\left(V_{n}\right)$ for any $n \geq 0$, then $\left\{V_{n}\right\}_{n>1}$ is an increasing sequence of finite subsets of $K$ and the closure of $V_{*}=\cup_{n \geq 1} V_{n}$ is $K$. For any $n \geq 1$, for any $w=w_{1} \ldots w_{n} \in W_{n}$, let

$$
V_{w}=f_{w_{1}} \circ \ldots \circ f_{w_{n}}\left(V_{0}\right)
$$

then $V_{n}=\cup_{w \in W_{n}} V_{w}$.

For any $n \geq 1$, for any $u \in l\left(V_{n}\right)$, let

$$
a_{n}(u)=\left(\frac{5}{3}\right)^{n} \sum_{w \in W_{n}} \sum_{p, q \in V_{w}}(u(p)-u(q))^{2},
$$

then for any $m \leq n$, for any $u \in l\left(V_{n}\right)$, we have $a_{m}(u) \leq a_{n}(u)$.

We have $\beta^{*}=\frac{\log 5}{\log 2}$ and $\left(\mathcal{E}_{\text {loc }}, \mathcal{F}_{\text {loc }}\right)$ on $L^{2}(K ; \nu)$ can be characterized as follows.

$$
\begin{aligned}
& \mathcal{E}_{\text {loc }}(u, u)=\lim _{n \rightarrow+\infty} a_{n}(u)=\sup _{n \geq 1} a_{n}(u), \\
& \mathcal{F}_{\text {loc }}=\left\{u \in C(K): \sup _{n \geq 1} a_{n}(u)<+\infty\right\} .
\end{aligned}
$$

We have the characterization of the domain of stable-like non-local Dirichlet form as follows, see [18, Theorem 1.1]. For any $\beta \in\left(\alpha, \beta^{*}\right)$, we have

$$
\mathcal{F}_{\beta}=\left\{u \in C(K): \sum_{n=1}^{+\infty} 2^{\left(\beta-\beta^{*}\right) n} a_{n}(u)<+\infty\right\} .
$$

Therefore, it is obvious that $\mathcal{F}_{\text {loc }} \subseteq \cap_{\beta \in\left(0, \beta^{*}\right)} \mathcal{F}_{\beta}$, to show that $\mathcal{F}_{\text {loc }} \varsubsetneqq \cap_{\beta \in\left(0, \beta^{*}\right)} \mathcal{F}_{\beta}$, we only need to construct $u \in C(K)$ such that $a_{n}(u)=n$ for any $n \geq 1$.

We construct $u \in l\left(V_{*}\right)$ by induction as follows.

For $n=1$. Let $u \in l\left(V_{1}\right)$ be given by $u=\frac{\sqrt{30}}{10} 1_{\left\{p_{0}\right\}}$, then $a_{1}(u)=1$.

Assume that we have constructed $u \in l\left(V_{n}\right)$ satisfying $a_{n}(u)=n$. Then for $n+1$, we only need to extend $u \in l\left(V_{n}\right)$ to a function on $V_{n+1}$ still denoted by $u \in l\left(V_{n+1}\right)$.

Recall that

$$
\begin{aligned}
a_{n+1}(u) & =\left(\frac{5}{3}\right)^{n+1} \sum_{w \in W_{n+1}} \sum_{p, q \in V_{w}}(u(p)-u(q))^{2} \\
& =\left(\frac{5}{3}\right)^{n+1} \sum_{w \in W_{n}}\left(\sum_{i \in W} \sum_{p, q \in V_{w i}}(u(p)-u(q))^{2}\right)
\end{aligned}
$$

For any $w \in W_{n}$, we only need to assign the values of $u$ on $\cup_{i \in W} V_{w i} \backslash V_{w}$, see Figure 2 .

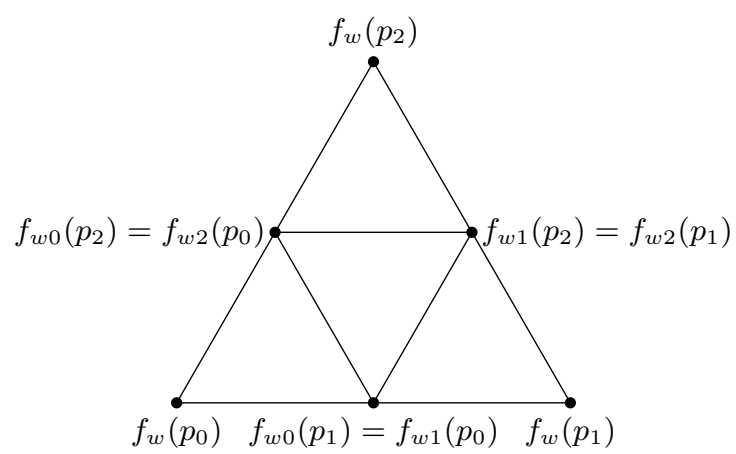

Figure 2: $\bigcup_{i \in W} V_{w i}$ 
Denote $a=u\left(f_{w}\left(p_{0}\right)\right), b=u\left(f_{w}\left(p_{1}\right)\right), c=u\left(f_{w}\left(p_{2}\right)\right)$, let

$$
\begin{aligned}
& u\left(f_{w 1}\left(p_{2}\right)\right)=x=\alpha b+\alpha c+(1-2 \alpha) a, \\
& u\left(f_{w 0}\left(p_{2}\right)\right)=y=\alpha c+\alpha a+(1-2 \alpha) b, \\
& u\left(f_{w 0}\left(p_{1}\right)\right)=z=\alpha a+\alpha b+(1-2 \alpha) c,
\end{aligned}
$$

where $\alpha \in\left(0, \frac{1}{2}\right)$ is some parameter, then

$$
\begin{aligned}
& \sum_{i \in W} \sum_{p, q \in V_{w i}}(u(p)-u(q))^{2} \\
& =(a-z)^{2}+(b-z)^{2}+(a-y)^{2}+(c-y)^{2}+(b-x)^{2}+(c-x)^{2} \\
& +(x-y)^{2}+(y-z)^{2}+(z-x)^{2} \\
& =\left(15 \alpha^{2}-12 \alpha+3\right)\left((a-b)^{2}+(b-c)^{2}+(c-a)^{2}\right) \\
& =\left(15 \alpha^{2}-12 \alpha+3\right) \sum_{p, q \in V_{w}}(u(p)-u(q))^{2} .
\end{aligned}
$$

Let $\varphi(\alpha)=15 \alpha^{2}-12 \alpha+3, \alpha \in\left(0, \frac{1}{2}\right)$, then

$$
\min _{\alpha \in\left(0, \frac{1}{2}\right)} \varphi(\alpha)=\varphi\left(\frac{2}{5}\right)=\frac{3}{5}, \lim _{\alpha \downarrow 0} \varphi(\alpha)=3, \lim _{\alpha \uparrow \frac{1}{2}} \varphi(\alpha)=\frac{3}{4},
$$

hence there exists $\alpha_{n} \in\left(0, \frac{2}{5}\right)$ such that $\varphi\left(\alpha_{n}\right)=\frac{3}{5} \frac{n+1}{n}$. Then we have the definition of $u$ on $\cup_{i \in W} V_{w i}$. Then we have the definition of $u$ on $V_{n+1}$. Moreover, $a_{n+1}(u)=\frac{n+1}{n} a_{n}(u)=n+1$.

By induction principle, we obtain $u \in l\left(V_{*}\right)$ satisfying $a_{n}(u)=n$ for any $n \geq 1$. Since $\alpha_{n} \uparrow$ $\frac{2}{5}$ as $n \rightarrow+\infty$, it is obvious that $u$ is uniformly continuous on $V_{*}$, hence $u$ can be extended to a continuous function on $K$ still denoted by $u \in C(K)$. Hence $u \in \cap_{\beta \in\left(0, \beta^{*}\right)} \mathcal{F}_{\beta} \backslash \mathcal{F}_{\text {loc }}$.

\section{Proof of Proposition 1.1 for the SC}

Let $K$ be the $\mathrm{SC}$ in $\mathbb{R}^{2}$, that is, let

$$
\begin{aligned}
& p_{0}=(0,0), p_{1}=\left(\frac{1}{2}, 0\right), p_{2}=(1,0), p_{3}=\left(1, \frac{1}{2}\right), \\
& p_{4}=(1,1), p_{5}=\left(\frac{1}{2}, 1\right), p_{6}=(0,1), p_{7}=\left(0, \frac{1}{2}\right),
\end{aligned}
$$

and

$$
f_{i}(x)=\frac{1}{3}\left(x-p_{i}\right)+p_{i}, x \in \mathbb{R}^{2}, i=0,1, \ldots, 7,
$$

then $K$ is the unique non-empty compact set in $\mathbb{R}^{2}$ satisfying $K=\cup_{i=0}^{7} f_{i}(K)$, see Figure 3 Let $|\cdot|$ be the Euclidean metric in $\mathbb{R}^{2}$ and $\nu$ the normalized Hausdorff measure of dimension $\alpha=\frac{\log 8}{\log 3}$ on $K$. Then $(K,|\cdot|, \nu)$ is an $\alpha$-regular compact metric measure space.

Denote $l(S)$ as the set of all real-valued functions on a set $S$.

We list the characterization of local regular Dirichlet form on the SC from 9 ] as follows, see also [1, 13, for reference.

Let $W_{0}=\{\emptyset\}$ and

$$
W_{n}=\left\{w=w_{1} \ldots w_{n}: w_{i}=0,1, \ldots, 7, i=1, \ldots, n\right\} \text { for any } n \geq 1 \text {. }
$$

For any $w^{(1)}=w_{1}^{(1)} \ldots w_{m}^{(1)} \in W_{m}, w^{(2)}=w_{1}^{(2)} \ldots w_{n}^{(2)} \in W_{n}$, let

$$
w^{(1)} w^{(2)}=w_{1}^{(1)} \ldots w_{m}^{(1)} w_{1}^{(2)} \ldots w_{n}^{(2)} \in W_{m+n} .
$$

For any $i=0,1, \ldots, 7$, let

$$
i^{n}=\underbrace{i \ldots i}_{n \text { times }} \in W_{n}
$$




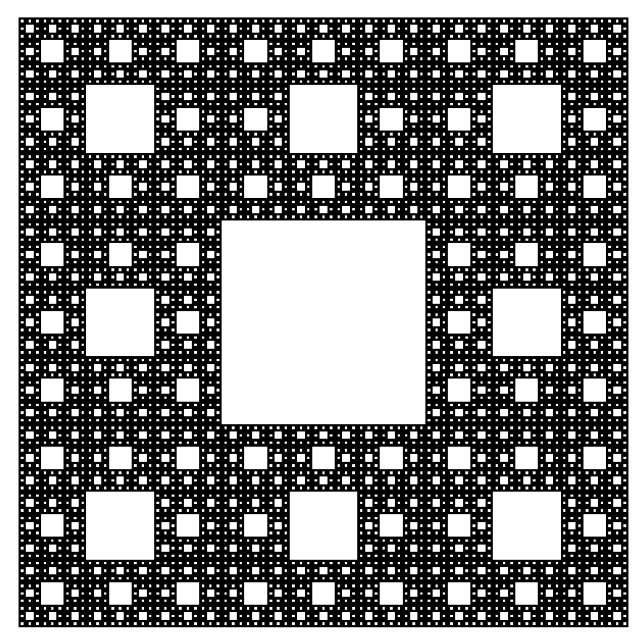

Figure 3: The SC in $\mathbb{R}^{2}$

Let $V_{0}=\left\{p_{0}, p_{1}, \ldots, p_{7}\right\}$ and $V_{n+1}=\cup_{i=0}^{7} f_{i}\left(V_{n}\right)$ for any $n \geq 0$, then $\left\{V_{n}\right\}_{n>0}$ is an increasing sequence of finite subsets of $K$ and the closure of $V_{*}=\cup_{n \geq 0} V_{n}$ is $K$. For any $n \geq 0$, for any $w=w_{1} \ldots w_{n} \in W_{n}$, let

$$
\begin{gathered}
f_{w}=f_{w_{1}} \circ \ldots \circ f_{w_{n}}, \\
V_{w}=f_{w}\left(V_{0}\right), K_{w}=f_{w}(K),
\end{gathered}
$$

then $V_{n}=\cup_{w \in W_{n}} V_{w}$. We use the convention that $f_{\emptyset}=\mathrm{Id}$ is the identity map.

Let $\rho$ be the parameter from resistance estimates in [2, 3, then

$$
\beta^{*}=\frac{\log (8 \rho)}{\log 3} .
$$

It was given in 2 , 3, that

- $\rho \in\left[\frac{7}{6}, \frac{3}{2}\right]$ based on shorting and cutting technique,

- $\rho \in[1.25147,1.25149]$ based on numerical calculation.

Hence $\rho>\frac{7}{6}$ which is a crucially important fact in the proof.

For any $n \geq 0$, for any $u \in l\left(V_{n}\right)$, let

$$
a_{n}(u)=\rho^{n} \sum_{w \in W_{n}} \sum_{\substack{p, q \in V_{w} \\|p-q|=2^{-1} \cdot 3^{-n}}}(u(p)-u(q))^{2} .
$$

By [9, Theorem 2.5], $\left(\mathcal{E}_{\text {loc }}, \mathcal{F}_{\text {loc }}\right)$ on $L^{2}(K ; \nu)$ can be characterized as follows.

$$
\begin{aligned}
& \mathcal{E}_{\text {loc }}(u, u) \asymp \sup _{n \geq 1} a_{n}(u), \\
& \mathcal{F}_{\text {loc }}=\left\{u \in C(K): \sup _{n \geq 1} a_{n}(u)<+\infty\right\} .
\end{aligned}
$$

We have the characterization of the domain of stable-like non-local Dirichlet form as follows, see [9, Lemma 2.1]. For any $\beta \in\left(\alpha, \beta^{*}\right)$, we have

$$
\mathcal{F}_{\beta}=\left\{u \in C(K): \sum_{n=1}^{+\infty} 3^{\left(\beta-\beta^{*}\right) n} a_{n}(u)<+\infty\right\} .
$$

Therefore, it is obvious that $\mathcal{F}_{\text {loc }} \subseteq \cap_{\beta \in\left(0, \beta^{*}\right)} \mathcal{F}_{\beta}$, to show that $\mathcal{F}_{\text {loc }} \varsubsetneqq \cap_{\beta \in\left(0, \beta^{*}\right)} \mathcal{F}_{\beta}$, we only need to prove the following result. 
Proposition 3.1. There exist $u \in C(K)$ and a universal positive constant $C$ satisfying $a_{n}(u) \leq C n$ for any $n \geq 1$ and there exists some sequence $\left\{n_{k}\right\}$ such that $a_{n_{k}}(u) \geq \frac{1}{C} n_{k}$ for any $k \geq 1$.

Proof of Proposition 1.1 using Proposition 3.1. Since $a_{n}(u) \leq C n$ for any $n \geq 1$, by Equation $(2)$, we have $u \in \mathcal{F}_{\beta}$ for any $\beta \in\left(\alpha, \beta^{*}\right)$. Since $a_{n_{k}}(u) \geq \frac{1}{C} n_{k}$ for any $k \geq 1$, by Equation [1], we have $u \notin \mathcal{F}_{\text {loc }}$. Hence $u \in \cap_{\beta \in\left(\alpha, \beta^{*}\right)} \mathcal{F}_{\beta} \backslash \mathcal{F}_{\text {loc }}=\cap_{\beta \in\left(0, \beta^{*}\right)} \mathcal{F}_{\beta} \backslash \mathcal{F}_{\text {loc }}$.

Denote

$$
L=(\{0,1\} \times[0,1]) \bigcup([0,1] \times\{0,1\}) .
$$

For any $n \geq 0$, for any $u \in l\left(V_{n}\right)$, denote

$$
E_{n}(u)=\sum_{w \in W_{n}} \sum_{\substack{p, q \in V_{w} \\|p-q|=2^{-1} \cdot 3^{-n}}}(u(p)-u(q))^{2} .
$$

We construct two functions in $C(K)$ that vanish on $L$ and have explicit asymptotic behaviours of $E_{n}$ as follows.

Lemma 3.2. There exist $u_{1} \in C(K)$ and a universal positive constant $C$ satisfying $0 \leq$ $u_{1} \leq 1$ on $K,\left.u_{1}\right|_{L}=0$ and

$$
\frac{1}{C}\left(\frac{1}{\rho}\right)^{n} \leq E_{n}\left(u_{1}\right) \leq C\left(\frac{1}{\rho}\right)^{n} \text { for any } n \geq 1
$$

Lemma 3.3. There exist $u_{2} \in C(K)$ and a universal positive constant $C$ satisfying $0 \leq$ $u_{2} \leq 1$ on $K,\left.u_{2}\right|_{L}=0$ and

$$
\frac{1}{C}\left(\frac{6}{7}\right)^{n} \leq E_{n}\left(u_{2}\right) \leq C\left(\frac{6}{7}\right)^{n} \text { for any } n \geq 1 .
$$

Remark 3.4. Since $\left.u_{1}\right|_{L}=\left.u_{2}\right|_{L}=0$, we have $E_{0}\left(u_{1}\right)=E_{0}\left(u_{2}\right)=0$. For any $n \geq 1$, the lower estimates of $E_{n}\left(u_{1}\right)$ and $E_{n}\left(u_{2}\right)$ imply that $u_{1}$ and $u_{2}$ are not identically zero on $V_{n}$.

Proof of Lemma 3.2. Denote

$$
S=\left(\left\{\frac{1}{3}, \frac{2}{3}\right\} \times\left[\frac{1}{3}, \frac{2}{3}\right]\right) \bigcup\left(\left[\frac{1}{3}, \frac{2}{3}\right] \times\left\{\frac{1}{3}, \frac{2}{3}\right\}\right) .
$$

For any $n \geq 1$, let

$$
R_{n}(S, L)=\inf \left\{E_{n}(u):\left.u\right|_{S \cap V_{n}}=1,\left.u\right|_{L \cap V_{n}}=0, u \in l\left(V_{n}\right)\right\}^{-1},
$$

then there exists a unique $u^{(n)} \in l\left(V_{n}\right)$ with $\left.u^{(n)}\right|_{S \cap V_{n}}=1,\left.u^{(n)}\right|_{L \cap V_{n}}=0$ such that $E_{n}\left(u^{(n)}\right)^{-1}=R_{n}(S, L)$. By flow technique and potential technique from [2, 3, 14, there exists a universal positive constant $C$ such that

$$
\frac{1}{C} \rho^{n} \leq R_{n}(S, L) \leq C \rho^{n} \text { for any } n \geq 1 .
$$

By the same proof as [9, Section 8] using uniform Harnack inequality, we obtain $u_{1} \in C(K)$ satisfying $0 \leq u_{1} \leq 1$ on $K,\left.u_{1}\right|_{S}=1,\left.u_{1}\right|_{L}=0$ and $a_{n}\left(u_{1}\right) \leq c C$ for any $n \geq 1$, where $c$ is the universal positive constant in weak monotonicity result [9, Theorem 7.1]. Hence

$$
E_{n}\left(u_{1}\right) \leq c C\left(\frac{1}{\rho}\right)^{n} \text { for any } n \geq 1
$$

By the optimal property of $u^{(n)}$, we have

$$
E_{n}\left(u_{1}\right) \geq E_{n}\left(u^{(n)}\right)=R_{n}(S, L)^{-1} \geq \frac{1}{C}\left(\frac{1}{\rho}\right)^{n} \text { for any } n \geq 1 .
$$


Proof of Lemma 3.3. Let $f \in C([0,1])$ be a strictly increasing function given by $f(0)=0$, $f(1)=1$ and for any $n \geq 0$, for any $i=0,1, \ldots, 3^{n}-1$

$$
\begin{aligned}
f\left(\frac{3 i+1}{3^{n+1}}\right) & =\frac{5}{7} f\left(\frac{i}{3^{n}}\right)+\frac{2}{7} f\left(\frac{i+1}{3^{n}}\right), \\
f\left(\frac{3 i+2}{3^{n+1}}\right) & =\frac{2}{7} f\left(\frac{i}{3^{n}}\right)+\frac{5}{7} f\left(\frac{i+1}{3^{n}}\right) .
\end{aligned}
$$

By the proof on [9, Page 4001], we have

$$
E_{n}((x, y) \mapsto f(x))=E_{n}((x, y) \mapsto f(y))=\left(\frac{6}{7}\right)^{n} \text { for any } n \geq 1,
$$

and for any $n \geq 1, f$ is the unique optimal function of the following variational problem

$$
\inf \left\{E_{n}((x, y) \mapsto f(x))=E_{n}((x, y) \mapsto f(y)): f(0)=0, f(1)=1, f \in C([0,1])\right\} .
$$

Let $u_{2} \in C(K)$ be given by $u_{2}(x, y)=f(x) f(1-x) f(y) f(1-y),(x, y) \in K$. It is obvious that $0 \leq u_{2} \leq 1$ on $K$ and $\left.u_{2}\right|_{L}=0$.

For any $n \geq 1$, by Minkowski inequality, we have

$$
\sqrt{E_{n}(u v)} \leq \max _{V_{n}}|u| \sqrt{E_{n}(v)}+\max _{V_{n}}|v| \sqrt{E_{n}(u)} \text { for any } u, v \in l\left(V_{n}\right),
$$

hence

$$
\sqrt{E_{n}\left(u_{2}\right)} \leq 4 \sqrt{E_{n}((x, y) \mapsto f(x))}
$$

hence

$$
E_{n}\left(u_{2}\right) \leq 16 E_{n}((x, y) \mapsto f(x))=16\left(\frac{6}{7}\right)^{n} \text { for any } n \geq 1 .
$$

For any $n \geq 1$, we have

$$
\begin{aligned}
& \geq \sum_{w \in W_{n}} \sum_{\substack{\left.p=\left(p_{x}, p_{y}\right), q=\left(q_{x}, q_{y}\right) \in V_{50 w} \\
|p-q|=-2-3-(n)+2\right) \\
p_{y}=q_{y}}}\left(f\left(p_{x}\right) f\left(1-p_{x}\right) f\left(p_{y}\right) f\left(1-p_{y}\right)\right. \\
& =\sum_{w \in W_{n}} \sum_{\substack{p=\left(p_{x}, p_{y}\right), q=\left(q_{x}, q_{y}\right) \in V_{50 w} \\
|p-q|=-1.3-(n+2) \\
p_{y}=q_{y}}}\left(f\left(p_{x}\right) f\left(1-p_{x}\right) f\left(1-q_{x}\right) f\left(q_{y}\right) f\left(1-q_{y}\right)\right)^{2}
\end{aligned}
$$

For any $p=\left(p_{x}, p_{y}\right) \in K_{50}$, we have $f\left(p_{y}\right) \in\left[\frac{5}{7}, \frac{39}{49}\right], f\left(1-p_{y}\right) \in\left[\frac{10}{49}, \frac{2}{7}\right]$. Hence

$$
\begin{aligned}
& E_{n+2}\left(u_{2}\right) \\
& \geq\left(\frac{5}{7}\right)^{2}\left(\frac{10}{49}\right)^{2} \sum_{w \in W_{n}} \sum_{\substack{p=\left(p_{x}, p_{y}\right), q=\left(q_{x}, q_{y}\right) \in V_{50 w} \\
|p-q|=2^{-1.3}-(n+2) \\
p_{y}=q_{y}}}\left(f\left(p_{x}\right) f\left(1-p_{x}\right)-f\left(q_{x}\right) f\left(1-q_{x}\right)\right)^{2} \\
& =\left(\frac{5}{7}\right)^{2}\left(\frac{10}{49}\right)^{2} \sum_{w \in W_{n}} \sum_{\substack{p=\left(p_{x}, p_{y}\right), q=\left(q_{x}, q_{y}\right) \in V_{50 w} \\
|p-q|=2^{-1}, 3^{-(n+2)}}}\left(f\left(p_{x}\right) f\left(1-p_{x}\right)-f\left(q_{x}\right) f\left(1-q_{x}\right)\right)^{2} \\
& =\left(\frac{5}{7}\right)^{2}\left(\frac{10}{49}\right)^{2} E_{n}\left(((x, y) \mapsto f(x) f(1-x)) \circ f_{50}\right) .
\end{aligned}
$$

Note that the function $((x, y) \mapsto f(x) f(1-x)) \circ f_{50} \in C(K)$ depends only on the first variable and maps 0 to $\frac{10}{49}$ and 1 to $\frac{580}{2401}$. By the optimal property of $f$, we have

$E_{n}\left(((x, y) \mapsto f(x) f(1-x)) \circ f_{50}\right) \geq\left(\frac{580}{2401}-\frac{10}{49}\right)^{2} E_{n}((x, y) \mapsto f(x))=\left(\frac{90}{2401}\right)^{2}\left(\frac{6}{7}\right)^{n}$ 
Hence

$$
E_{n+2}\left(u_{2}\right) \geq\left(\frac{5}{7}\right)^{2}\left(\frac{10}{49}\right)^{2}\left(\frac{90}{2401}\right)^{2}\left(\frac{6}{7}\right)^{n}=\frac{562500}{13841287201}\left(\frac{6}{7}\right)^{n+2} \text { for any } n \geq 1
$$

Hence

$$
E_{n}\left(u_{2}\right) \geq C\left(\frac{6}{7}\right)^{n} \text { for any } n \geq 1
$$

where $C=\min \left\{\frac{7}{6} E_{1}\left(u_{2}\right),\left(\frac{7}{6}\right)^{2} E_{2}\left(u_{2}\right), \frac{562500}{13841287201}\right\}$ is some universal positive constant.

We only need to prove Proposition 3.1 as follows.

Proof of Proposition 3.1. In terms of $E_{n}$, we need to construct $u \in C(K)$ with a universal positive constant $C$ satisfying $E_{n}(u) \leq C n\left(\frac{1}{\rho}\right)^{n}$ for any $n \geq 1$ and there exists some sequence $\left\{n_{k}\right\}$ such that $E_{n_{k}}(u) \geq \frac{1}{C} n_{k}\left(\frac{1}{\rho}\right)^{n_{k}}$ for any $k \geq 1$.

The idea of the proof is to use the fact that $\rho>\frac{7}{6}$ which implies that $\left(\frac{6}{7}\right)^{n}$ is much larger than $n\left(\frac{1}{\rho}\right)^{n}$ for any sufficiently large $n \geq 1$ and the fact that $n\left(\frac{1}{\rho}\right)^{n}$ is much larger than $\left(\frac{1}{\rho}\right)^{n}$ for any sufficiently large $n \geq 1$.

For any $A \subseteq K$, for any $n \geq 1$, for any $u \in l\left(V_{n}\right)$, denote

$$
E_{n}^{A}(u)=\sum_{\substack{w \in W_{n} \\ K_{w} \subseteq A}} \sum_{\substack{p, q \in V_{w} \\|p-q|=2^{-1} \cdot 3^{-n}}}(u(p)-u(q))^{2} .
$$

Let $C_{1}$ be the universal positive constant from Lemma 3.2 For simplicity, we may assume that $C_{1}$ is an integer. Take arbitrary integer $C \geq C_{1}+1$.

Let $u^{(1)}=\delta_{2} u_{2}$, where $\delta_{2}$ is some positive parameter to be determined, then

$$
E_{n}\left(u^{(1)}\right)=\delta_{2}^{2} E_{n}\left(u_{2}\right) \text { for any } n \geq 1 .
$$

Take $\delta_{2}>0$ such that

$$
E_{n}\left(u^{(1)}\right)=\delta_{2}^{2} E_{n}\left(u_{2}\right)<C n\left(\frac{1}{\rho}\right)^{n} \text { for any } n=1,2 .
$$

By Lemma 3.3 , there exists $n_{1}>2$ such that

$$
\begin{aligned}
& E_{n}\left(u^{(1)}\right)<C n\left(\frac{1}{\rho}\right)^{n} \text { for any } n=1, \ldots, n_{1}-1, \\
& E_{n_{1}}\left(u^{(1)}\right) \geq C n_{1}\left(\frac{1}{\rho}\right)^{n_{1}} .
\end{aligned}
$$

Define $u^{(2)} \in l(K)$ as follows. For any $w \in W_{n_{1}-1}$, let

$$
\left.u^{(2)}\right|_{K_{w}}= \begin{cases}\delta_{1} u_{1} \circ f_{w}, & \text { if } w \neq 0^{n_{1}-1} \\ \delta_{2} u_{2} \circ f_{w}, & \text { if } w=0^{n_{1}-1}\end{cases}
$$

where $\delta_{1}, \delta_{2}$ are some positive parameters to be determined. Since $\left.u_{1}\right|_{L}=\left.u_{2}\right|_{L}=0$, we have $u^{(2)} \in C(K)$ is well-defined and

$$
E_{n}\left(u^{(2)}\right)= \begin{cases}0, & \text { if } n=1, \ldots, n_{1}-1 \\ \delta_{1}^{2}\left(8^{n_{1}-1}-1\right) E_{n-n_{1}+1}\left(u_{1}\right)+\delta_{2}^{2} E_{n-n_{1}+1}\left(u_{2}\right), & \text { if } n=n_{1}, n_{1}+1, \ldots\end{cases}
$$

Take $\delta_{1} \in(0,+\infty)$ such that

$$
\delta_{1}^{2}\left(8^{n_{1}-1}-1\right) E_{1}\left(u_{1}\right)=\frac{1}{2 C} n_{1}\left(\frac{1}{\rho}\right)^{n_{1}},
$$


then

$$
\delta_{1}=\sqrt{\frac{\frac{1}{2 C} n_{1}\left(\frac{1}{\rho}\right)^{n_{1}}}{\left(8^{n_{1}-1}-1\right) E_{1}\left(u_{1}\right)}} \leq \sqrt{\frac{n_{1}\left(\frac{1}{\rho}\right)^{n_{1}}}{14 C E_{1}\left(u_{1}\right)}} .
$$

By Lemma 3.2 , we have

$$
\begin{aligned}
& \delta_{1}^{2}\left(8^{n_{1}-1}-1\right) E_{n-n_{1}+1}\left(u_{1}\right) \leq C_{1}^{2}\left(\frac{1}{\rho}\right)^{n-n_{1}} \delta_{1}^{2}\left(8^{n_{1}-1}-1\right) E_{1}\left(u_{1}\right) \\
& =\frac{C_{1}^{2}}{2 C} n_{1}\left(\frac{1}{\rho}\right)^{n} \leq \frac{C_{1}^{2}}{2 C} n\left(\frac{1}{\rho}\right)^{n}<C n\left(\frac{1}{\rho}\right)^{n} \text { for any } n=n_{1}, n_{1}+1, \ldots
\end{aligned}
$$

Take $\delta_{2} \in(0,1)$ such that

$$
\begin{gathered}
E_{n}\left(u^{(2)}\right)=\delta_{1}^{2}\left(8^{n_{1}-1}-1\right) E_{n-n_{1}+1}\left(u_{1}\right)+\delta_{2}^{2} E_{n-n_{1}+1}\left(u_{2}\right)<C n\left(\frac{1}{\rho}\right)^{n} \\
\text { for any } n=n_{1}, n_{1}+1, \ldots, 2 C_{1}^{2} C^{2} n_{1}, 2 C_{1}^{2} C^{2} n_{1}+1 .
\end{gathered}
$$

Hence

$$
\begin{gathered}
E_{n}\left(u^{(2)}\right)<C n\left(\frac{1}{\rho}\right)^{n} \text { for any } n=1, \ldots, 2 C_{1}^{2} C^{2} n_{1}, 2 C_{1}^{2} C^{2} n_{1}+1, \\
E_{n_{1}}^{K \backslash K_{0^{n_{1}}-1}}\left(u^{(2)}\right) \geq \frac{1}{2 C} n_{1}\left(\frac{1}{\rho}\right)^{n_{1}} .
\end{gathered}
$$

Assume that we have constructed $u^{(k)} \in C(K)$ and $n_{k-1}>\ldots>n_{1}>2$ satisfying that $\left.u^{(k)}\right|_{K_{0^{n} n^{-1}}}$ is the product of some positive parameter and $u_{2} \circ f_{0^{n_{k-1}-1}},\left.u^{(k)}\right|_{K \backslash K_{0^{n} n_{-1}-1}}$ is constructed by gluing the terms of the form $\delta u_{1} \circ f_{w}$ with $\delta \in(0,+\infty)$ and $w \in W_{1} \cup \ldots \cup$ $W_{n_{k-1}-1}$, and

$$
\begin{gathered}
E_{n}\left(u^{(k)}\right)<C n\left(\frac{1}{\rho}\right)^{n} \text { for any } n=1, \ldots, 2 C_{1}^{2} C^{2} n_{k-1}, 2 C_{1}^{2} C^{2} n_{k-1}+1, \\
E_{n_{i}}^{K \backslash K_{0^{n_{k-1}-1}}}\left(u^{(k)}\right) \geq \frac{1}{2 C} n_{i}\left(\frac{1}{\rho}\right)^{n_{i}} \text { for any } i=1, \ldots, k-1 .
\end{gathered}
$$

By Lemma 3.3 , there exists $n_{k}>2 C_{1}^{2} C^{2} n_{k-1}+1$ such that

$$
\begin{aligned}
E_{n}\left(u^{(k)}\right) & <C n\left(\frac{1}{\rho}\right)^{n} \text { for any } n=1, \ldots, n_{k}-1, \\
E_{n_{k}}\left(u^{(k)}\right) & \geq C n_{k}\left(\frac{1}{\rho}\right)^{n_{k}}
\end{aligned}
$$

Define $u^{(k+1)} \in l(K)$ as follows. Let $\left.u^{(k+1)}\right|_{K \backslash K_{0} n_{k-1}-1}=\left.u^{(k)}\right|_{K \backslash K_{0^{n_{k-1}-1}}}$ and for any $w \in W_{n_{k}-n_{k-1}}$, let

$$
\left.u^{(k+1)}\right|_{K_{0^{n_{k-1}-1} w}}= \begin{cases}\delta_{1} u_{1} \circ f_{0^{n_{k-1}-1}}, & \text { if } w \neq 0^{n_{k}-n_{k-1}}, \\ \delta_{2} u_{2} \circ f_{0^{n_{k-1}-1} w}, & \text { if } w=0^{n_{k}-n_{k-1}},\end{cases}
$$

where $\delta_{1}, \delta_{2}$ are some positive parameters to be determined, see Figure 4

Since $\left.u_{1}\right|_{L}=\left.u_{2}\right|_{L}=0$, we have $u^{(k+1)} \in C(K)$ is well-defined, $\left.u^{(k+1)}\right|_{K_{0^{n}-1}-1}$ is the product of some positive parameter and $u_{2} \circ f_{0^{n_{k}-1}},\left.u^{(k+1)}\right|_{K \backslash K_{0^{n} k^{-1}}}$ is constructed by gluing the terms of the form $\delta u_{1} \circ f_{w}$ with $\delta \in(0,+\infty)$ and $w \in W_{1} \cup \ldots \cup W_{n_{k}-1}$, and

$$
\begin{aligned}
& E_{n}\left(u^{(k+1)}\right) \\
& =\left\{\begin{array}{cc}
E_{n}^{K \backslash K_{0^{n_{k-1}-1}}}\left(u^{(k)}\right), & \text { if } n=1, \ldots, n_{k}-1, \\
E_{n}^{K \backslash K_{0^{n} k^{-1}-1}}\left(u^{(k)}\right)+\delta_{1}^{2}\left(8^{n_{k}-n_{k-1}}-1\right) E_{n-n_{k}+1}\left(u_{1}\right) & \\
+\delta_{2}^{2} E_{n-n_{k}+1}\left(u_{2}\right), & \text { if } n=n_{k}, n_{k}+1, \ldots
\end{array}\right.
\end{aligned}
$$




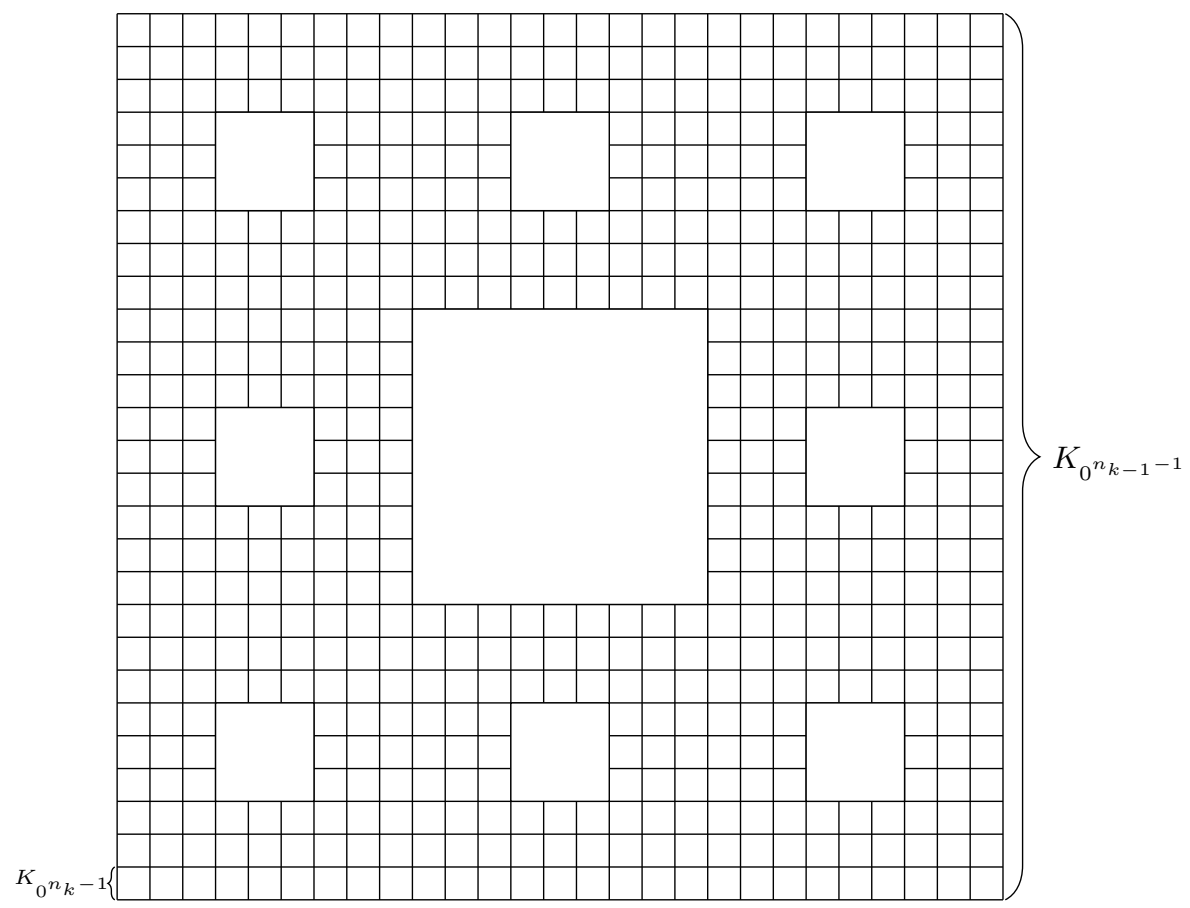

Figure 4: The Construction of $u^{(k+1)}$

Hence

$$
\begin{gathered}
E_{n}\left(u^{(k+1)}\right)=E_{n}^{K \backslash K_{0^{n} n^{-1}}}\left(u^{(k)}\right) \leq E_{n}\left(u^{(k)}\right)<C n\left(\frac{1}{\rho}\right)^{n} \text { for any } n=1, \ldots, n_{k}-1, \\
E_{n_{i}}^{K \backslash K_{0^{n} n_{k}-1}}\left(u^{(k+1)}\right) \geq E_{n_{i}}^{K \backslash K_{0^{n_{k-1}-1}}}\left(u^{(k+1)}\right)=E_{n_{i}}^{K \backslash K_{0} n_{k-1}-1}\left(u^{(k)}\right) \geq \frac{1}{2 C} n_{i}\left(\frac{1}{\rho}\right)^{n_{i}} \\
\text { for any } i=1, \ldots, k-1 .
\end{gathered}
$$

Since $\left.u^{(k+1)}\right|_{K \backslash K_{0^{n} k_{-1}-1}}=\left.u^{(k)}\right|_{K \backslash K_{0} n_{k-1}-1}$ is constructed by gluing the terms of the form $\delta u_{1} \circ f_{w}$ with $\delta \in(0,+\infty)$ and $w \in W_{1} \cup \ldots \cup W_{n_{k-1}-1}$, by Lemma 3.2 we have

$$
\begin{aligned}
& E_{n}^{K \backslash K_{0} n_{k-1}-1}\left(u^{(k)}\right) \leq C_{1}^{2}\left(\frac{1}{\rho}\right)^{n-n_{k-1}} E_{n_{k-1}}^{K \backslash K_{0^{n} n_{k-1}-1}}\left(u^{(k)}\right) \leq C_{1}^{2}\left(\frac{1}{\rho}\right)^{n-n_{k-1}} E_{n_{k-1}}\left(u^{(k)}\right) \\
& <C_{1}^{2}\left(\frac{1}{\rho}\right)^{n-n_{k-1}} C n_{k-1}\left(\frac{1}{\rho}\right)^{n_{k-1}}=C_{1}^{2} C n_{k-1}\left(\frac{1}{\rho}\right)^{n} \text { for any } n=n_{k-1}, n_{k-1}+1, \ldots
\end{aligned}
$$

Hence

$$
\begin{aligned}
E_{n}^{K \backslash K_{0} n_{k-1}-1}\left(u^{(k)}\right) & <C_{1}^{2} C n_{k-1}\left(\frac{1}{\rho}\right)^{n}<\frac{1}{2 C} n\left(\frac{1}{\rho}\right)^{n} \\
& \text { for any } n=2 C_{1}^{2} C^{2} n_{k-1}+1,2 C_{1}^{2} C^{2} n_{k-1}+2, \ldots
\end{aligned}
$$

In particular

$$
E_{n_{k}}^{K \backslash K_{0^{n_{k-1}-1}}}\left(u^{(k)}\right)<\frac{1}{2 C} n_{k}\left(\frac{1}{\rho}\right)^{n_{k}} .
$$

Take $\delta_{1} \in(0,+\infty)$ such that

$$
E_{n_{k}}^{K \backslash K_{0^{n_{k}-1}}}\left(u^{(k+1)}\right)=E_{n_{k}}^{K \backslash K_{0} n_{k-1}-1}\left(u^{(k)}\right)+\delta_{1}^{2}\left(8^{n_{k}-n_{k-1}}-1\right) E_{1}\left(u_{1}\right)=\frac{1}{2 C} n_{k}\left(\frac{1}{\rho}\right)^{n_{k}},
$$

then

$$
\delta_{1} \leq \sqrt{\frac{\frac{1}{2 C} n_{k}\left(\frac{1}{\rho}\right)^{n_{k}}}{\left(8^{n_{k}-n_{k-1}}-1\right) E_{1}\left(u_{1}\right)}} \leq \sqrt{\frac{n_{k}\left(\frac{1}{\rho}\right)^{n_{k}}}{14 C E_{1}\left(u_{1}\right)}}
$$


Since $\left.u^{(k+1)}\right|_{K \backslash K_{0^{n_{k-1}-1}}}=\left.u^{(k)}\right|_{K \backslash K_{0^{n_{k-1}-1}}}$ is constructed by gluing the terms of the form $\delta u_{1} \circ f_{w}$ with $\delta \in(0,+\infty)$ and $w \in W_{1} \cup \ldots \cup W_{n_{k-1}-1}$, by Lemma 3.2, we have

$$
\begin{aligned}
& E_{n}^{K \backslash K_{0^{n_{k-1}-1}}}\left(u^{(k)}\right)+\delta_{1}^{2}\left(8^{n_{k}-n_{k-1}}-1\right) E_{n-n_{k}+1}\left(u_{1}\right) \\
& \leq C_{1}^{2}\left(\frac{1}{\rho}\right)^{n-n_{k}}\left(E_{n_{k}}^{K \backslash K_{0} n_{k-1}-1}\left(u^{(k)}\right)+\delta_{1}^{2}\left(8^{n_{k}-n_{k-1}}-1\right) E_{1}\left(u_{1}\right)\right) \\
& =\frac{C_{1}^{2}}{2 C} n_{k}\left(\frac{1}{\rho}\right)^{n} \leq \frac{C_{1}^{2}}{2 C} n\left(\frac{1}{\rho}\right)^{n}<C n\left(\frac{1}{\rho}\right)^{n} \text { for any } n=n_{k}, n_{k}+1, \ldots
\end{aligned}
$$

Take $\delta_{2} \in\left(0, \frac{1}{k^{2}}\right)$ such that

$$
\begin{aligned}
E_{n}\left(u^{(k+1)}\right) & =E_{n}^{K \backslash K_{0^{n_{k-1}-1}}}\left(u^{(k)}\right)+\delta_{1}^{2}\left(8^{n_{k}-n_{k-1}}-1\right) E_{n-n_{k}+1}\left(u_{1}\right)+\delta_{2}^{2} E_{n-n_{k}+1}\left(u_{2}\right) \\
& <C n\left(\frac{1}{\rho}\right)^{n} \text { for any } n=n_{k}, n_{k}+1, \ldots, 2 C_{1}^{2} C^{2} n_{k}, 2 C_{1}^{2} C^{2} n_{k}+1 .
\end{aligned}
$$

Therefore, we have

$$
\begin{gathered}
E_{n}\left(u^{(k+1)}\right)<C n\left(\frac{1}{\rho}\right)^{n} \text { for any } n=1, \ldots, 2 C_{1}^{2} C^{2} n_{k}, 2 C_{1}^{2} C^{2} n_{k}+1, \\
E_{n_{i}}^{K \backslash K_{0^{n} k^{-1}}}\left(u^{(k+1)}\right) \geq \frac{1}{2 C} n_{i}\left(\frac{1}{\rho}\right)^{n_{i}} \text { for any } i=1, \ldots, k .
\end{gathered}
$$

By induction principle, we obtain $\left\{u^{(k)}\right\} \subseteq C(K)$.

By construction, for any $k \geq 2$, we have

$$
\begin{aligned}
& \left.u^{(k)}\right|_{K \backslash K_{0} n_{k-1}-1}=\left.u^{(k+1)}\right|_{K \backslash K_{0} n_{k-1}-1}, \\
& 0 \leq\left. u^{(k)}\right|_{K_{0^{n} k-1-1}} \leq \frac{1}{(k-1)^{2}}, \\
& 0 \leq\left. u^{(k+1)}\right|_{K_{0} n_{k-1}-1} \leq \sqrt{\frac{n_{k}\left(\frac{1}{\rho}\right)^{n_{k}}}{14 C E_{1}\left(u_{1}\right)}}+\frac{1}{k^{2}},
\end{aligned}
$$

hence

$$
\sup _{K}\left|u^{(k)}-u^{(k+1)}\right| \leq \sqrt{\frac{n_{k}\left(\frac{1}{\rho}\right)^{n_{k}}}{14 C E_{1}\left(u_{1}\right)}}+\frac{1}{k^{2}}+\frac{1}{(k-1)^{2}} .
$$

Hence for any $k>l \geq 2$, we have

$$
\sup _{K}\left|u^{(k)}-u^{(l)}\right| \leq \sum_{i=l}^{k-1} \sup _{K}\left|u^{(i)}-u^{(i+1)}\right| \leq \sum_{i=l}^{k-1}\left(\sqrt{\frac{n_{i}\left(\frac{1}{\rho}\right)^{n_{i}}}{14 C E_{1}\left(u_{1}\right)}}+\frac{1}{i^{2}}+\frac{1}{(i-1)^{2}}\right) \rightarrow 0
$$

as $k, l \rightarrow+\infty$. Hence $\left\{u^{(k)}\right\} \subseteq C(K)$ converges uniformly to some function $u \in C(K)$.

For any fixed $n \geq 1$, there exists $l \geq 1$ such that $n \leq 2 C_{1}^{2} C^{2} n_{k}+1$ for any $k \geq l$, hence

$$
E_{n}\left(u^{(k+1)}\right)<C n\left(\frac{1}{\rho}\right)^{n} \text { for any } k \geq l .
$$

Letting $k \rightarrow+\infty$, we have

$$
E_{n}(u)=\lim _{k \rightarrow+\infty} E_{n}\left(u^{(k+1)}\right) \leq C n\left(\frac{1}{\rho}\right)^{n} \text { for any } n \geq 1 .
$$

For any fixed $i \geq 1$, for any $k \geq i$, we have

$$
E_{n_{i}}\left(u^{(k+1)}\right) \geq E_{n_{i}}^{K \backslash K_{0^{n} k-1}}\left(u^{(k+1)}\right) \geq \frac{1}{2 C} n_{i}\left(\frac{1}{\rho}\right)^{n_{i}} .
$$

Letting $k \rightarrow+\infty$, we have

$$
E_{n_{i}}(u)=\lim _{k \rightarrow+\infty} E_{n_{i}}\left(u^{(k+1)}\right) \geq \frac{1}{2 C} n_{i}\left(\frac{1}{\rho}\right)^{n_{i}} \text { for any } i \geq 1 .
$$

Therefore, $u \in C(K)$ is our desired function. 


\section{Proof of Theorem 1.5}

First, we list some results about closed form.

Let $(\mathcal{E}, \mathcal{F})$ on $L^{2}(K ; \nu)$ be a closed form that corresponds to a strongly continuous semi-group $\left\{T_{t}: t>0\right\}$ on $L^{2}(K ; \nu)$. By [6. Section 1.3], there exists a spectral family $\left\{E_{\lambda}: \lambda \in[0,+\infty)\right\}$ such that

$$
\begin{aligned}
& \mathcal{E}(u, u)=\int_{[0,+\infty)} \lambda \mathrm{d}\left(E_{\lambda} u, u\right), \\
& \mathcal{F}=\left\{u \in L^{2}(K ; \nu): \int_{[0,+\infty)} \lambda \mathrm{d}\left(E_{\lambda} u, u\right)<+\infty\right\},
\end{aligned}
$$

and

$$
T_{t}=\int_{[0,+\infty)} e^{-t \lambda} \mathrm{d} E_{\lambda} \text { for any } t \in(0,+\infty) .
$$

For any $t \in(0,+\infty)$, for any $u \in L^{2}(K ; \nu)$, let

$$
\mathcal{E}_{(t)}(u, u)=\frac{1}{t}\left(u-T_{t} u, u\right)
$$

We have the following result.

Lemma 4.1. ([6, Lemma 1.3.4]) For any $u \in L^{2}(K ; \nu)$, we have $t \mapsto \mathcal{E}_{(t)}(u, u)$ is monotone decreasing in $(0,+\infty)$ and

$$
\begin{aligned}
& \mathcal{E}(u, u)=\lim _{t \downarrow 0} \mathcal{E}_{(t)}(u, u), \\
& \mathcal{F}=\left\{u \in L^{2}(K ; \nu): \lim _{t \downarrow 0} \mathcal{E}_{(t)}(u, u)<+\infty\right\} .
\end{aligned}
$$

Second, we give some results about subordinated Hunt process.

Let $(\mathcal{E}, \mathcal{F})$ be a regular Dirichlet form on $L^{2}(K ; \nu)$ that corresponds to a Hunt process $\left\{X_{t}\right\}$. For any $\delta \in(0,1)$, let $\left\{\xi_{t}^{(\delta)}\right\}$ be the $\delta$-stable subordinator, that is, the one-dimensional Lévy process whose Laplace transform is given by $\mathbb{E} e^{-s \xi_{t}^{(\delta)}}=e^{-t s^{\delta}}$, let $\eta_{t}^{(\delta)}$ be its onedimensional distribution density. Assume that the processes $\left\{X_{t}\right\}$ and $\left\{\xi_{t}^{(\delta)}\right\}$ are independent, then the $\delta$-subordinated Hunt process $\left\{X_{t}^{(\delta)}\right\}$ is given by $\left\{X_{\xi_{t}^{(\delta)}}\right\}$. Let $\left\{T_{t}^{(\delta)}: t>0\right\}$ on $L^{2}(K ; \nu)$ be the strongly continuous Markovian semi-group and $\left(\mathcal{E}^{(\delta)}, \mathcal{F}^{(\delta)}\right)$ on $L^{2}(K ; \nu)$ the regular Dirichlet form that correspond to the Hunt process $\left\{X_{t}^{(\delta)}\right\}$, then

$$
T_{t}^{(\delta)} u=\int_{0}^{+\infty} T_{s} u\left(\eta_{t}^{(\delta)}(s) \mathrm{d} s\right) \text { for any } t \in(0,+\infty), \text { for any } u \in L^{2}(K ; \nu),
$$

see [5, 16].

We have the characterization of $\mathcal{E}^{(\delta)}$ as follows.

Proposition 4.2. For any $\delta \in(0,1)$, for any $u \in L^{2}(K ; \nu)$, we have

$$
\mathcal{E}^{(\delta)}(u, u)=\int_{[0,+\infty)} \lambda^{\delta} \mathrm{d}\left(E_{\lambda} u, u\right)=\frac{\delta}{\Gamma(1-\delta)} \int_{0}^{+\infty} \frac{1}{s^{\delta}} \mathcal{E}_{(s)}(u, u) \mathrm{d} s(\leq+\infty)
$$

Remarks 4.3. (1) For any $u \in L^{2}(K ; \nu) \cdot t^{-1}\left(u-T_{t}^{(\delta)} u, u\right)$ is non-negative finite for any $t \in(0,+\infty)$, monotone decreasing in $t \in(0,+\infty)$ and $\mathcal{E}^{(\delta)}(u, u)$ is defined as its limit as $t \downarrow 0$ by Lemma 4.1 which is allowed to be $+\infty . \mathrm{d}\left(E_{\lambda} u, u\right)$ is an ordinary measure on $[0,+\infty)$ and $\mathcal{E}_{(s)}(u, u)$ is non-negative finite for any $s \in(0,+\infty)$, hence the above two integrals are well-defined and allowed also to be $+\infty$.

(2) [15, Equation (3.5)] gave the above second equality only for any $u \in \mathcal{F}$ where the condition $u \in \mathcal{F}$ is intrinsically used to apply dominated convergence theorem. 
Proof. For any $u \in L^{2}(K ; \nu)$, by Lemma 4.1 and Equation 3 , we have

$$
\begin{aligned}
& \mathcal{E}^{(\delta)}(u, u)=\lim _{t \downarrow 0} \frac{1}{t}\left(u-T_{t}^{(\delta)} u, u\right) \\
& =\lim _{t \downarrow 0} \frac{1}{t}\left((u, u)-\int_{0}^{+\infty}\left(T_{s} u, u\right) \eta_{t}^{(\delta)}(s) \mathrm{d} s\right) \\
& =\lim _{t \downarrow 0} \frac{1}{t} \int_{0}^{+\infty}\left(u-T_{s} u, u\right) \eta_{t}^{(\delta)}(s) \mathrm{d} s \\
& =\lim _{t \downarrow 0} \frac{1}{t} \int_{0}^{+\infty}\left(\int_{[0,+\infty)}\left(1-e^{-s \lambda}\right) \mathrm{d}\left(E_{\lambda} u, u\right)\right) \eta_{t}^{(\delta)}(s) \mathrm{d} s \\
& =\lim _{t \downarrow 0} \frac{1}{t} \int_{[0,+\infty)}\left(\int_{0}^{+\infty}\left(1-e^{-s \lambda}\right) \eta_{t}^{(\delta)}(s) \mathrm{d} s\right) \mathrm{d}\left(E_{\lambda} u, u\right) \\
& =\lim _{t \downarrow 0} \frac{1}{t} \int_{[0,+\infty)}\left(1-e^{-t \lambda^{\delta}}\right) \mathrm{d}\left(E_{\lambda} u, u\right) .
\end{aligned}
$$

Since $t \mapsto \frac{1-e^{-t \lambda^{\delta}}}{t}$ is monotone decreasing in $(0,+\infty)$ for any $\lambda \in[0,+\infty)$, by monotone convergence theorem, we have

$$
\begin{aligned}
& \mathcal{E}^{(\delta)}(u, u)=\lim _{t \downarrow 0} \frac{1}{t} \int_{[0,+\infty)}\left(1-e^{-t \lambda^{\delta}}\right) \mathrm{d}\left(E_{\lambda} u, u\right) \\
& =\int_{[0,+\infty)} \lim _{t \downarrow 0} \frac{1-e^{-t \lambda^{\delta}}}{t} \mathrm{~d}\left(E_{\lambda} u, u\right)=\int_{[0,+\infty)} \lambda^{\delta} \mathrm{d}\left(E_{\lambda} u, u\right) .
\end{aligned}
$$

Recall that for any $\delta \in(0,1)$, we have

$$
\int_{0}^{+\infty} \frac{1-e^{-s}}{s^{1+\delta}} \mathrm{d} s=\frac{\Gamma(1-\delta)}{\delta}
$$

which implies that for any $\lambda \in[0,+\infty)$, we have

$$
\int_{0}^{+\infty} \frac{1-e^{-s \lambda}}{s^{1+\delta}} \mathrm{d} s=\frac{\Gamma(1-\delta)}{\delta} \lambda^{\delta} .
$$

Hence

$$
\begin{aligned}
& \mathcal{E}^{(\delta)}(u, u)=\int_{[0,+\infty)} \lambda^{\delta} \mathrm{d}\left(E_{\lambda} u, u\right)=\frac{\delta}{\Gamma(1-\delta)} \int_{[0,+\infty)}\left(\int_{0}^{+\infty} \frac{1-e^{-s \lambda}}{s^{1+\delta}} \mathrm{d} s\right) \mathrm{d}\left(E_{\lambda} u, u\right) \\
& =\frac{\delta}{\Gamma(1-\delta)} \int_{0}^{+\infty} \frac{1}{s^{1+\delta}}\left(\int_{[0,+\infty)}\left(1-e^{-s \lambda}\right) \mathrm{d}\left(E_{\lambda} u, u\right)\right) \mathrm{d} s \\
& =\frac{\delta}{\Gamma(1-\delta)} \int_{0}^{+\infty} \frac{1}{s^{1+\delta}}\left(u-T_{s} u, u\right) \mathrm{d} s=\frac{\delta}{\Gamma(1-\delta)} \int_{0}^{+\infty} \frac{1}{s^{\delta}} \mathcal{E}_{(s)}(u, u) \mathrm{d} s .
\end{aligned}
$$

We have some direct corollaries as follows.

\section{Corollary 4.4.}

(1) For any $\delta \in(0,1)$, we have

$$
\begin{aligned}
\mathcal{F}^{(\delta)} & =\left\{u \in L^{2}(K ; \nu): \int_{[0,+\infty)} \lambda^{\delta} \mathrm{d}\left(E_{\lambda} u, u\right)<+\infty\right\} \\
& =\left\{u \in L^{2}(K ; \nu): \int_{(1,+\infty)} \lambda^{\delta} \mathrm{d}\left(E_{\lambda} u, u\right)<+\infty\right\} .
\end{aligned}
$$

(2) For any $\delta_{1}, \delta_{2} \in(0,1)$ with $\delta_{1}<\delta_{2}$, we have $\mathcal{F}^{\left(\delta_{1}\right)} \supseteq \mathcal{F}^{\left(\delta_{2}\right)} \supseteq \mathcal{F}$. 
Proof. (1) The first equality follows directly from Proposition 4.2 Since for any $u \in$ $L^{2}(K ; \nu)$, we have

$$
\int_{[0,1]} \lambda^{\delta} \mathrm{d}\left(E_{\lambda} u, u\right) \leq \int_{[0,1]} \mathrm{d}\left(E_{\lambda} u, u\right) \leq \int_{[0,+\infty)} \mathrm{d}\left(E_{\lambda} u, u\right)=(u, u)<+\infty .
$$

Hence

$$
\int_{[0,+\infty)} \lambda^{\delta} \mathrm{d}\left(E_{\lambda} u, u\right)<+\infty
$$

if and only if

$$
\int_{(1,+\infty)} \lambda^{\delta} \mathrm{d}\left(E_{\lambda} u, u\right)<+\infty
$$

Hence we have the second equality.

(2) It follows easily from (1).

Corollary 4.5. For any $\delta \in(0,1)$.

(1) $\mathcal{F}$ is $\left(\mathcal{E}^{(\delta)}(\cdot, \cdot)+(\cdot, \cdot)\right)$-dense in $\mathcal{F}^{(\delta)}$.

(2) Any core of $(\mathcal{E}, \mathcal{F})$ on $L^{2}(K ; \nu)$ is a core of $\left(\mathcal{E}^{(\delta)}, \mathcal{F}^{(\delta)}\right)$ on $L^{2}(K ; \nu)$.

Remark 4.6. If $\left(\mathcal{E}^{(\delta)}, \mathcal{F}^{(\delta)}\right)$ on $L^{2}(K ; \nu)$ is defined only as the Dirichlet form corresponding to the strongly continuous Markovian semi-group $\left\{T_{t}^{(\delta)}: t>0\right\}$ on $L^{2}(K ; \nu)$ which is given by Equation (3), then the regular property of $\left(\mathcal{E}^{(\delta)}, \mathcal{F}^{(\delta)}\right)$ on $L^{2}(K ; \nu)$ follows also from the regular property of $(\mathcal{E}, \mathcal{F})$ on $L^{2}(K ; \nu)$ and the above result.

Proof. (1) For any $t \in(0,+\infty)$, for any $u \in L^{2}(K ; \nu)$, we claim that $T_{t}^{(\delta)} u \in \mathcal{F}$. We only need to show that

$$
\int_{[0,+\infty)} \lambda \mathrm{d}\left(E_{\lambda} T_{t}^{(\delta)} u, T_{t}^{(\delta)} u\right)<+\infty .
$$

Indeed

$$
\begin{aligned}
& \int_{[0,+\infty)} \lambda \mathrm{d}\left(E_{\lambda} T_{t}^{(\delta)} u, T_{t}^{(\delta)} u\right)=\int_{[0,+\infty)} \int_{0}^{+\infty} \int_{0}^{+\infty} \lambda \eta_{t}^{(\delta)}(r) \eta_{t}^{(\delta)}(s) \mathrm{d}\left(E_{\lambda} T_{r} u, T_{s} u\right) \mathrm{d} r \mathrm{~d} s \\
& =\int_{[0,+\infty)} \lambda\left(\int_{0}^{+\infty} \int_{0}^{+\infty} e^{-r \lambda} e^{-s \lambda} \eta_{t}^{(\delta)}(r) \eta_{t}^{(\delta)}(s) \mathrm{d} r \mathrm{~d} s\right) \mathrm{d}\left(E_{\lambda} u, u\right) \\
& =\int_{[0,+\infty)} \lambda e^{-2 t \lambda^{\delta}} \mathrm{d}\left(E_{\lambda} u, u\right) .
\end{aligned}
$$

Since $\lambda \mapsto \lambda e^{-2 t \lambda^{\delta}}$ is continuous on $[0,+\infty)$ and $\lim _{\lambda \rightarrow+\infty} \lambda e^{-2 t \lambda^{\delta}}=0$, there exists some positive constant $C$ such that

$$
0 \leq \lambda e^{-2 t \lambda^{\delta}} \leq C \text { for any } \lambda \in[0,+\infty) .
$$

Hence

$$
\int_{[0,+\infty)} \lambda \mathrm{d}\left(E_{\lambda} T_{t}^{(\delta)} u, T_{t}^{(\delta)} u\right)=\int_{[0,+\infty)} \lambda e^{-2 t \lambda^{\delta}} \mathrm{d}\left(E_{\lambda} u, u\right) \leq C(u, u)<+\infty .
$$

For any $u \in \mathcal{F}^{(\delta)}$, by [6, Lemma 1.3 .3 (iii)], we have $T_{t}^{(\delta)} u \in \mathcal{F}$ is $\left(\mathcal{E}^{(\delta)}(\cdot, \cdot)+(\cdot, \cdot)\right)$ convergent to $u$ as $t \downarrow 0$. Hence $\mathcal{F}$ is $\left(\mathcal{E}^{(\delta)}(\cdot, \cdot)+(\cdot, \cdot)\right)$-dense in $\mathcal{F}^{(\delta)}$.

(2) For any $u \in \mathcal{F}$, by Proposition 4.2 we have

$$
\begin{aligned}
\mathcal{E}^{(\delta)}(u, u) & =\int_{[0,1]} \lambda^{\delta} \mathrm{d}\left(E_{\lambda} u, u\right)+\int_{(1,+\infty)} \lambda^{\delta} \mathrm{d}\left(E_{\lambda} u, u\right) \\
& \leq \int_{[0,1]} \mathrm{d}\left(E_{\lambda} u, u\right)+\int_{(1,+\infty)} \lambda \mathrm{d}\left(E_{\lambda} u, u\right) \\
& \leq \int_{[0,+\infty)} \mathrm{d}\left(E_{\lambda} u, u\right)+\int_{[0,+\infty)} \lambda \mathrm{d}\left(E_{\lambda} u, u\right)
\end{aligned}
$$




$$
=\mathcal{E}(u, u)+(u, u)
$$

Hence

$$
\mathcal{E}^{(\delta)}(u, u)+(u, u) \leq 2(\mathcal{E}(u, u)+(u, u)) \text { for any } u \in \mathcal{F} .
$$

Let $\mathcal{C}$ be a core of $(\mathcal{E}, \mathcal{F})$ on $L^{2}(K ; \nu)$, that is, $\mathcal{C}$ is $(\mathcal{E}(\cdot, \cdot)+(\cdot, \cdot))$-dense in $\mathcal{F}$ and uniformly dense in $C_{c}(K)$. We only need to show that $\mathcal{C}$ is $\left(\mathcal{E}^{(\delta)}(\cdot, \cdot)+(\cdot, \cdot)\right)$-dense in $\mathcal{F}^{(\delta)}$. Indeed, by the above inequality, we have $\mathcal{C}$ is $\left(\mathcal{E}^{(\delta)}(\cdot, \cdot)+(\cdot, \cdot)\right)$-dense in $\mathcal{F}$. Since $\mathcal{F}$ is $\left(\mathcal{E}^{(\delta)}(\cdot, \cdot)+(\cdot, \cdot)\right)$ dense in $\mathcal{F}^{(\delta)}$ which is $(1)$, we have $\mathcal{C}$ is $\left(\mathcal{E}^{(\delta)}(\cdot, \cdot)+(\cdot, \cdot)\right)$-dense in $\mathcal{F}^{(\delta)}$.

Corollary 4.7. Let $p_{t}(x, \mathrm{~d} y)$ be the transition density of the regular Dirichlet form $(\mathcal{E}, \mathcal{F})$ on $L^{2}(K ; \nu)$. Then for any $\delta \in(0,1)$, we have

$$
\mathcal{E}^{(\delta)}(u, u)=\frac{1}{2} \int_{K} \int_{K}(u(x)-u(y))^{2} J^{(\delta)}(\mathrm{d} x \mathrm{~d} y)+\int_{K} u(x)^{2} k^{(\delta)}(\mathrm{d} x) \text { for any } u \in L^{2}(K ; \nu),
$$

where

$$
\begin{aligned}
J^{(\delta)}(\mathrm{d} x \mathrm{~d} y) & =\frac{\delta}{\Gamma(1-\delta)} \int_{0}^{+\infty} \frac{1}{s^{1+\delta}} p_{s}(x, \mathrm{~d} y) \nu(\mathrm{d} x) \mathrm{d} s \\
k^{(\delta)}(\mathrm{d} x) & =\frac{\delta}{\Gamma(1-\delta)} \int_{0}^{+\infty} \frac{1}{s^{1+\delta}}\left(1-\int_{K} p_{s}(x, \mathrm{~d} y)\right) \nu(\mathrm{d} x) \mathrm{d} s .
\end{aligned}
$$

Remark 4.8. The above result is indeed the Beurling-Deny decomposition of the regular Dirichlet form $\left(\mathcal{E}^{(\delta)}, \mathcal{F}^{(\delta)}\right)$ on $L^{2}(K ; \nu)$ which has only jumping part and killing part, see [6, Theorem 3.2.1, Lemma 4.5.4]. Hence $\left(\mathcal{E}^{(\delta)}, \mathcal{F}^{(\delta)}\right)$ on $L^{2}(K ; \nu)$ is always non-local.

Proof. For any $u \in L^{2}(K ; \nu)$, for any $s \in(0,+\infty)$, we have

$$
\left(u-T_{s} u, u\right)=\frac{1}{2} \int_{K} \int_{K}(u(x)-u(y))^{2} p_{s}(x, \mathrm{~d} y) \nu(\mathrm{d} x)+\int_{K} u(x)^{2}\left(1-\int_{K} p_{s}(x, \mathrm{~d} y)\right) \nu(\mathrm{d} x) .
$$

Then the result follows directly from Proposition 4.2

Third, we give the proof of Theorem 1.5 as follows.

Proof of Theorem 1.5. It follows directly from Corollary $4.4(2)$ that $\mathcal{F} \subseteq \cap_{\delta \in(0,1)} \mathcal{F}^{(\delta)}$. If $A$ is bounded, then

$$
L^{2}(K ; \nu)=\mathcal{D}(A) \subseteq \mathcal{D}(\sqrt{-A})=\mathcal{F} \subseteq \bigcap_{\delta \in(0,1)} \mathcal{F}^{(\delta)} \subseteq L^{2}(K ; \nu),
$$

hence we have "=". We only need to show that if $A$ is unbounded, then " $\subsetneq$ " holds.

Let $E$ be the spectral measure corresponding to the spectral family $\left\{E_{\lambda}: \lambda \in[0,+\infty)\right\}$, that is,

$$
E\left(\left(\lambda_{1}, \lambda_{2}\right]\right)=E_{\lambda_{2}}-E_{\lambda_{1}} \text { for any } \lambda_{1}, \lambda_{2} \in[0,+\infty) \text { with } \lambda_{1}<\lambda_{2} .
$$

Then $\left\{E\left(\left(2^{k}, 2^{k+1}\right]\right)\right\}_{k \geq 0}$ is a sequence of orthogonal projections on $L^{2}(K ; \nu)$ satisfying

$$
E\left(\left(2^{k}, 2^{k+1}\right]\right) E\left(\left(2^{l}, 2^{l+1}\right]\right)=0 \text { for any } k, l \geq 0 \text { with } k \neq l .
$$

Let

$$
I=\left\{k \geq 0: E\left(\left(2^{k}, 2^{k+1}\right]\right) \neq 0\right\} .
$$

Since $A$ is unbounded, the spectrum $\sigma(-A) \subseteq[0,+\infty)$ is unbounded, $\# I=+\infty$.

For any $k \in I$, there exists $u_{k} \in E\left(\left(2^{k}, 2^{k+1}\right]\right)\left(L^{2}(K ; \nu)\right)$ with $\left\|u_{k}\right\|_{L^{2}(K ; \nu)}^{2}=2^{-(k+1)}$. For any $k \notin I$, let $u_{k}=0$. Then $\left(u_{k}, u_{l}\right)=0$ for any $k, l \geq 0$ with $k \neq l$.

Let $u=\sum_{k=0}^{\infty} u_{k}$. Then $u \in L^{2}(K ; \nu)$ and

$$
\|u\|_{L^{2}(K ; \nu)}^{2}=\sum_{k=0}^{\infty}\left\|u_{k}\right\|_{L^{2}(K ; \nu)}^{2} \leq \sum_{k=0}^{\infty} \frac{1}{2^{k+1}}=1 .
$$


For any $k \geq 0$, we have $E\left(\left(2^{k}, 2^{k+1}\right]\right) u=u_{k}$. Hence

$$
\begin{aligned}
& \int_{(1,+\infty)} \lambda \mathrm{d}\left(E_{\lambda} u, u\right)=\sum_{k=0}^{\infty} \int_{\left(2^{k}, 2^{k+1}\right]} \lambda \mathrm{d}\left(E_{\lambda} u, u\right) \geq \sum_{k \in I} \int_{\left(2^{k}, 2^{k+1}\right]} \lambda \mathrm{d}\left(E_{\lambda} u, u\right) \\
& \geq \sum_{k \in I} \int_{\left(2^{k}, 2^{k+1}\right]} 2^{k} \mathrm{~d}\left(E_{\lambda} u, u\right)=\sum_{k \in I} 2^{k}\left(E\left(\left(2^{k}, 2^{k+1}\right]\right) u, u\right)=\sum_{k \in I} 2^{k}\left(u_{k}, u\right) \\
& =\sum_{k \in I} 2^{k}\left\|u_{k}\right\|_{L^{2}(K ; \nu)}^{2}=\sum_{k \in I} 2^{k} \frac{1}{2^{k+1}}=\frac{1}{2} \# I=+\infty
\end{aligned}
$$

hence $u \notin \mathcal{F}$.

For any $\delta \in(0,1)$, we have

$$
\begin{aligned}
& \int_{(1,+\infty)} \lambda^{\delta} \mathrm{d}\left(E_{\lambda} u, u\right)=\sum_{k=0}^{\infty} \int_{\left(2^{k}, 2^{k+1}\right]} \lambda^{\delta} \mathrm{d}\left(E_{\lambda} u, u\right) \leq \sum_{k=0}^{\infty} \int_{\left(2^{k}, 2^{k+1}\right]} 2^{\delta(k+1)} \mathrm{d}\left(E_{\lambda} u, u\right) \\
& =\sum_{k=0}^{\infty} 2^{\delta(k+1)}\left(E\left(\left(2^{k}, 2^{k+1}\right]\right) u, u\right)=\sum_{k=0}^{\infty} 2^{\delta(k+1)}\left(u_{k}, u\right)=\sum_{k=0}^{\infty} 2^{\delta(k+1)}\left\|u_{k}\right\|_{L^{2}(K ; \nu)}^{2} \\
& \leq \sum_{k=0}^{\infty} 2^{\delta(k+1)} \frac{1}{2^{k+1}}=\sum_{k=0}^{\infty} \frac{1}{2^{(1-\delta)(k+1)}}<+\infty,
\end{aligned}
$$

hence by Corollary $4.4(1)$, we have $u \in \mathcal{F}^{(\delta)}$ for any $\delta \in(0,1)$.

Therefore, we have $u \in \cap_{\delta \in(0,1)} \mathcal{F}^{(\delta)} \backslash \mathcal{F}$.

\section{Proof of Proposition 1.3}

By Theorem 1.5. we only need to show that the generator $A$ of $(\mathcal{E}, \mathcal{F})$ on $L^{2}(K ; \nu)$ is unbounded and that $\mathcal{F}^{(\delta)}=\mathcal{F}_{\delta \beta_{0}}$ for any $\delta \in(0,1)$.

First, we have the following result.

Lemma 5.1. Let $(K, d, \nu)$ be an $\alpha$-regular metric measure space. Let $(\mathcal{E}, \mathcal{F})$ be a conservative regular Dirichlet form on $L^{2}(K ; \nu)$ with a heat kernel $p_{t}(x, y)$ satisfying

$$
p_{t}(x, y) \geq \frac{C_{1}}{t^{\alpha / \beta_{0}}} \Phi\left(C_{2} \frac{d(x, y)}{t^{1 / \beta_{0}}}\right)
$$

for any $x, y \in K$, for any $t \in\left(0, \operatorname{diam}(K)^{\beta_{0}}\right)$, here $\operatorname{diam}(K)=\sup \{d(x, y): x, y \in K\}$ is infinite if $K$ is unbounded and is finite if $K$ is bounded, where $\beta_{0} \in(0,+\infty)$ is some parameter, $C_{1}, C_{2}$ are some positive constants and $\Phi:(0,+\infty) \rightarrow(0,+\infty)$ is some monotone decreasing function. Then the generator $A$ of $(\mathcal{E}, \mathcal{F})$ on $L^{2}(K ; \nu)$ is unbounded.

Remark 5.2. The boundedness of the generator is sensitive to the small scale behaviours of the space and the heat kernel. For example, on $\mathbb{Z}$, the generator of the standard random walk is bounded with spectrum $[-2,0]$, but on $\mathbb{R}$, the generator of the standard Brownian motion is unbounded with spectrum $(-\infty, 0]$.

Proof. Since $(\mathcal{E}, \mathcal{F})$ on $L^{2}(K ; \nu)$ is conservative, we have

$$
\int_{K} p_{t}(x, y) \nu(\mathrm{d} y)=1 \text { for any } t \in(0,+\infty) \text {, for any } x \in K .
$$

Then for any $u \in L^{2}(K ; \nu)$, we have

$$
\mathcal{E}(u, u)=\lim _{t \downarrow 0} \frac{1}{t}\left(u-T_{t} u, u\right)=\lim _{t \downarrow 0} \frac{1}{2 t} \int_{K} \int_{K}(u(x)-u(y))^{2} p_{t}(x, y) \nu(\mathrm{d} y) \nu(\mathrm{d} x),
$$

where $t \mapsto \frac{1}{2 t} \int_{K} \int_{K} \ldots \nu(\mathrm{d} y) \nu(\mathrm{d} x)$ is monotone decreasing in $(0,+\infty)$. Hence for any $r \in$ $(0, \operatorname{diam}(K))$, letting $t=r^{\beta_{0}}$, we have

$$
\mathcal{E}(u, u) \geq \frac{1}{2 t} \int_{K} \int_{B(x, r)}(u(x)-u(y))^{2} p_{t}(x, y) \nu(\mathrm{d} y) \nu(\mathrm{d} x)
$$




$$
\begin{aligned}
& \geq \frac{1}{2 t} \int_{K} \int_{B(x, r)}(u(x)-u(y))^{2} \frac{C_{1}}{t^{\alpha / \beta_{0}}} \Phi\left(C_{2} \frac{d(x, y)}{t^{1 / \beta_{0}}}\right) \nu(\mathrm{d} y) \nu(\mathrm{d} x) \\
& \geq \frac{1}{2 t} \int_{K} \int_{B(x, r)}(u(x)-u(y))^{2} \frac{C_{1}}{t^{\alpha / \beta_{0}}} \Phi\left(C_{2} \frac{r}{t^{1 / \beta_{0}}}\right) \nu(\mathrm{d} y) \nu(\mathrm{d} x) \\
& =\frac{C_{1} \Phi\left(C_{2}\right)}{2} \frac{1}{r^{\alpha+\beta_{0}}} \int_{K} \int_{B(x, r)}(u(x)-u(y))^{2} \nu(\mathrm{d} y) \nu(\mathrm{d} x) .
\end{aligned}
$$

Suppose that $A$ is bounded, then $\sqrt{-A}$ is also bounded, hence $\mathcal{F}=\mathcal{D}(\sqrt{-A})=L^{2}(K ; \nu)$ and

$$
\mathcal{E}(u, u)=(\sqrt{-A} u, \sqrt{-A} u) \leq\|\sqrt{-A}\|^{2}\|u\|_{L^{2}(K ; \nu)}^{2}=\|A\|\|u\|_{L^{2}(K ; \nu)}^{2} \text { for any } u \in L^{2}(K ; \nu) .
$$

Hence

$$
\begin{aligned}
& \frac{C_{1} \Phi\left(C_{2}\right)}{2} \frac{1}{r^{\alpha+\beta_{0}}} \int_{K} \int_{B(x, r)}(u(x)-u(y))^{2} \nu(\mathrm{d} y) \nu(\mathrm{d} x) \\
& \leq\|A\|\|u\|_{L^{2}(K ; \nu)}^{2} \text { for any } u \in L^{2}(K ; \nu) \text {, for any } r \in(0, \operatorname{diam}(K)) .
\end{aligned}
$$

Since $K$ is $\alpha$-regular, there exists some positive constant $C$ such that

$$
\frac{1}{C} r^{\alpha} \leq \nu(B(x, r)) \leq C r^{\alpha} \text { for any } x \in K \text {, for any } r \in(0, \operatorname{diam}(K)) \text {. }
$$

Let $c:=2 C^{2 / \alpha} \geq 2$. We claim that

$$
B(x, c r) \backslash B(x, r) \neq \emptyset \text { for any } x \in K, \text { for any } r \in\left(0, \frac{1}{c} \operatorname{diam}(K)\right) .
$$

Indeed, we have

$$
\nu(B(x, c r)) \geq \frac{1}{C}(c r)^{\alpha}=2^{\alpha} C r^{\alpha}>C r^{\alpha} \geq \nu(B(x, r)),
$$

hence $B(x, c r) \backslash B(x, r) \neq \emptyset$.

For any $r \in\left(0, \operatorname{diam}(K) /\left(c^{3}+2\right)\right)$, take arbitrary $x_{0} \in K$, let $u=1_{B\left(x_{0}, r\right)}$, then RHS of Equation $(5)=\|A\| \nu\left(B\left(x_{0}, r\right)\right) \lesssim r^{\alpha}$.

On the other hand

$$
\begin{aligned}
\text { LHS of Equation } \sqrt{5]} & \gtrsim \frac{1}{\left(\left(c^{3}+2\right) r\right)^{\alpha+\beta_{0}}} \int_{K} \int_{B\left(x,\left(c^{3}+2\right) r\right)}(u(x)-u(y))^{2} \nu(\mathrm{d} y) \nu(\mathrm{d} x) \\
& \asymp \frac{1}{r^{\alpha+\beta_{0}}} \int_{B\left(x_{0}, r\right)} \int_{B\left(x,\left(c^{3}+2\right) r\right) \backslash B\left(x_{0}, r\right)}(1-0)^{2} \nu(\mathrm{d} y) \nu(\mathrm{d} x) \\
& =\frac{1}{r^{\alpha+\beta_{0}}} \int_{B\left(x_{0}, r\right)} \nu\left(B\left(x,\left(c^{3}+2\right) r\right) \backslash B\left(x_{0}, r\right)\right) \nu(\mathrm{d} x) \\
& \geq \frac{1}{r^{\alpha+\beta_{0}}} \int_{B\left(x_{0}, r\right)} \nu\left(B\left(x_{0}, c^{3} r\right) \backslash B\left(x_{0}, r\right)\right) \nu(\mathrm{d} x) \\
& =\frac{1}{r^{\alpha+\beta_{0}}} \nu\left(B\left(x_{0}, c^{3} r\right) \backslash B\left(x_{0}, r\right)\right) \nu\left(B\left(x_{0}, r\right)\right) .
\end{aligned}
$$

Since $B\left(x_{0}, c^{2} r\right) \backslash B\left(x_{0}, c r\right) \neq \emptyset$, taking arbitrary $z \in B\left(x_{0}, c^{2} r\right) \backslash B\left(x_{0}, c r\right)$, we have

$$
B(z, r) \subseteq B\left(x_{0}, c^{3} r\right) \backslash B\left(x_{0}, r\right),
$$

then

$$
\nu\left(B\left(x_{0}, c^{3} r\right) \backslash B\left(x_{0}, r\right)\right) \geq \nu(B(z, r)) .
$$

Hence

LHS of Equation $(5) \gtrsim \frac{1}{r^{\alpha+\beta_{0}}} \nu(B(z, r)) \nu\left(B\left(x_{0}, r\right)\right) \gtrsim \frac{1}{r^{\alpha+\beta_{0}}} \cdot r^{\alpha} \cdot r^{\alpha}=r^{\alpha-\beta_{0}}$.

Therefore, we have

$$
r^{\alpha-\beta_{0}} \lesssim r^{\alpha} \text { for any } r \in\left(0, \frac{\operatorname{diam}(K)}{c^{3}+2}\right)
$$

Letting $r \downarrow 0$, we obtain a contradiction! Hence $A$ is unbounded. 
Second, we show that $\mathcal{F}^{(\delta)}=\mathcal{F}_{\delta \beta_{0}}$ for any $\delta \in(0,1)$. Indeed, the calculation of jumping kernels from heat kernels by subordination is standard. We give the calculation here for completeness.

Proof of $\mathcal{F}^{(\delta)}=\mathcal{F}_{\delta \beta_{0}}$ for any $\delta \in(0,1)$. By Corollary 4.7 , we have

$$
\begin{aligned}
& \mathcal{E}^{(\delta)}(u, u)=\frac{1}{2} \int_{K} \int_{K}(u(x)-u(y))^{2} J^{(\delta)}(x, y) \nu(\mathrm{d} x) \nu(\mathrm{d} y), \\
& \mathcal{F}^{(\delta)}=\left\{u \in L^{2}(K ; \nu): \int_{K} \int_{K}(u(x)-u(y))^{2} J^{(\delta)}(x, y) \nu(\mathrm{d} x) \nu(\mathrm{d} y)<+\infty\right\},
\end{aligned}
$$

where

$$
J^{(\delta)}(x, y)=\frac{\delta}{\Gamma(1-\delta)} \int_{0}^{+\infty} \frac{1}{t^{1+\delta}} p_{t}(x, y) \mathrm{d} t .
$$

Hence, we only need to show that

$$
J^{(\delta)}(x, y) \asymp \frac{1}{d(x, y)^{\alpha+\delta \beta_{0}}} .
$$

By [7, Theorem 4.1], we have the following dichotomy.

(a) Either $(\mathcal{E}, \mathcal{F})$ on $L^{2}(K ; \nu)$ is local, $\beta_{0} \in[2, \alpha+1]$ and

$$
\Phi(s) \asymp C \exp \left(-c s^{\frac{\beta_{0}}{\beta_{0}-1}}\right) .
$$

(b) Or $(\mathcal{E}, \mathcal{F})$ on $L^{2}(K ; \nu)$ is non-local, $\beta_{0} \in(0, \alpha+1]$ and

$$
\Phi(s) \asymp(1+s)^{-\left(\alpha+\beta_{0}\right)} .
$$

For (a). We have

$$
\frac{C_{1}}{t^{\alpha / \beta_{0}}} \exp \left(-C_{2}\left(\frac{d(x, y)}{t^{1 / \beta_{0}}}\right)^{\frac{\beta_{0}}{\beta_{0}-1}}\right) \leq p_{t}(x, y) \leq \frac{C_{3}}{t^{\alpha / \beta_{0}}} \exp \left(-C_{4}\left(\frac{d(x, y)}{t^{1 / \beta_{0}}}\right)^{\frac{\beta_{0}}{\beta_{0}-1}}\right)
$$

for any $x, y \in K$, for any $t \in\left(0, \operatorname{diam}(K)^{\beta_{0}}\right)$.

Note the following elementary results. For any $a \in(1,+\infty), b, c, d \in(0,+\infty)$, we have

$$
\begin{aligned}
\int_{0}^{+\infty} \frac{1}{t^{a}} \exp \left(-\frac{c}{t^{b}}\right) \mathrm{d} t & =\frac{\Gamma\left(\frac{a-1}{b}\right)}{b c^{\frac{a-1}{b}}} \\
\int_{0}^{d} \frac{1}{t^{a}} \exp \left(-\frac{c}{t^{b}}\right) \mathrm{d} t & =\frac{1}{b c^{\frac{a-1}{b}}} \int_{\frac{c}{d^{b}}}^{+\infty} s^{\frac{a-1}{b}-1} e^{-s} \mathrm{~d} s .
\end{aligned}
$$

If $\operatorname{diam}(K)=+\infty$. By Equation (6), we have

$$
\begin{aligned}
& \delta(1-\delta)\left[\left(\beta_{0}-1\right) \frac{\Gamma\left(\frac{\left(\beta_{0}-1\right)\left(\alpha+\delta \beta_{0}\right)}{\beta_{0}}\right)}{\Gamma(2-\delta)} \frac{C_{1}}{\left.C_{2}^{\frac{\left(\beta_{0}-1\right)\left(\alpha+\delta \beta_{0}\right)}{\beta_{0}}}\right]}\right] \frac{1}{d(x, y)^{\alpha+\delta \beta_{0}}} \leq J^{(\delta)}(x, y) \\
& \leq \delta(1-\delta)\left[\left(\beta_{0}-1\right) \frac{\Gamma\left(\frac{\left(\beta_{0}-1\right)\left(\alpha+\delta \beta_{0}\right)}{\beta_{0}}\right)}{\Gamma(2-\delta)} \frac{C_{3}}{C_{4}^{\frac{\left(\beta_{0}-1\right)\left(\alpha+\delta \beta_{0}\right)}{\beta_{0}}}}\right] \frac{1}{d(x, y)^{\alpha+\delta \beta_{0}}} \text { for any } x, y \in K .
\end{aligned}
$$

If $\operatorname{diam}(K)<+\infty$. Using semi-group property, we have

$$
p_{t}(x, y) \leq \frac{C_{3}}{\operatorname{diam}(K)^{\alpha}} \text { for any } t \in\left[\operatorname{diam}(K)^{\beta_{0}},+\infty\right) \text {, for any } x, y \in K .
$$

By Equation (6), we have

$$
J^{(\delta)}(x, y)=\frac{\delta}{\Gamma(1-\delta)}\left(\int_{0}^{\operatorname{diam}(K)^{\beta_{0}}}+\int_{\operatorname{diam}(K)^{\beta_{0}}}^{+\infty}\right) \frac{1}{t^{1+\delta}} p_{t}(x, y) \mathrm{d} t
$$




$$
\begin{aligned}
& \leq \frac{\delta}{\Gamma(1-\delta)}\left(\int_{0}^{\operatorname{diam}(K)^{\beta_{0}}} \frac{1}{t^{1+\delta}} \frac{C_{3}}{t^{\alpha / \beta_{0}}} \exp \left(-C_{4}\left(\frac{d(x, y)}{t^{1 / \beta_{0}}}\right)^{\frac{\beta_{0}}{\beta_{0}-1}}\right) \mathrm{d} t\right. \\
& \left.+\int_{\operatorname{diam}(K)^{\beta_{0}}}^{+\infty} \frac{1}{t^{1+\delta}} \frac{C_{3}}{\operatorname{diam}(K)^{\alpha}} \mathrm{d} t\right) \\
& \leq \frac{\delta}{\Gamma(1-\delta)}\left(\int_{0}^{+\infty} \frac{1}{t^{1+\delta}} \frac{C_{3}}{t^{\alpha / \beta_{0}}} \exp \left(-C_{4}\left(\frac{d(x, y)}{t^{1 / \beta_{0}}}\right)^{\frac{\beta_{0}}{\beta_{0}-1}}\right) \mathrm{d} t+\frac{1}{\delta} C_{3} \frac{1}{\operatorname{diam}(K)^{\alpha+\delta \beta_{0}}}\right) \\
& \leq(1-\delta)\left[\frac{C_{3}}{\Gamma(2-\delta)}\left(\delta\left(\beta_{0}-1\right) \frac{\Gamma\left(\frac{\left(\beta_{0}-1\right)\left(\alpha+\delta \beta_{0}\right)}{\beta_{0}}\right)}{C_{4}^{\frac{\left(\beta_{0}-1\right)\left(\alpha+\beta_{0}\right)}{\beta_{0}}}}+1\right)\right] \frac{1}{d(x, y)^{\alpha+\delta \beta_{0}}} .
\end{aligned}
$$

On the other hand, by Equation (7), we have

$$
\begin{aligned}
& J^{(\delta)}(x, y) \geq \frac{\delta}{\Gamma(1-\delta)} \int_{0}^{\operatorname{diam}(K)^{\beta_{0}}} \frac{1}{t^{1+\delta}} p_{t}(x, y) \mathrm{d} t \\
& \geq \frac{\delta}{\Gamma(1-\delta)} \int_{0}^{\operatorname{diam}(K)^{\beta_{0}}} \frac{1}{t^{1+\delta}} \frac{C_{1}}{t^{\alpha / \beta_{0}}} \exp \left(-C_{2}\left(\frac{d(x, y)}{t^{1 / \beta_{0}}}\right)^{\frac{\beta_{0}}{\beta_{0}-1}}\right) \mathrm{d} t \\
& =\frac{\delta}{\Gamma(1-\delta)} C_{1} \frac{\beta_{0}-1}{C_{2}^{\frac{\left(\beta_{0}-1\right)\left(\alpha+\delta \beta_{0}\right)}{\beta_{0}}} d(x, y)^{\alpha+\delta \beta_{0}}} \int_{C_{2}\left(\frac{d(x, y)}{\operatorname{diam}(K)}\right)^{\frac{\beta_{0}}{\beta_{0}-1}} t^{\frac{\left(\beta_{0}-1\right)\left(\alpha+\delta \beta_{0}\right)}{\beta_{0}}-1} e^{-t} \mathrm{~d} t}^{+\infty} \int_{C_{2}}^{+\infty} t^{\frac{\left(\beta_{0}-1\right)\left(\alpha+\delta \beta_{0}\right)}{\beta_{0}}-1} e^{-t} \mathrm{~d} t \\
& \left.\geq \frac{\delta}{\Gamma(1-\delta)} C_{1} \frac{\beta_{0}-1}{C_{2}^{\frac{\left(\beta_{0}-1\right)\left(\alpha+\delta \beta_{0}\right)}{\beta_{0}}} d(x, y)^{\alpha+\delta \beta_{0}}} \int_{C_{2}}^{+\infty} t^{\frac{\left(\beta_{0}-1\right)\left(\alpha+\delta \beta_{0}\right)}{\beta_{0}}-1} e^{-t} \mathrm{~d} t\right] \frac{1}{d(x, y)^{\alpha+\delta \beta_{0}}} .
\end{aligned}
$$

For (b). We have

$$
\frac{C_{1}}{t^{\alpha / \beta_{0}}}\left(1+\frac{d(x, y)}{t^{1 / \beta_{0}}}\right)^{-\left(\alpha+\beta_{0}\right)} \leq p_{t}(x, y) \leq \frac{C_{2}}{t^{\alpha / \beta_{0}}}\left(1+\frac{d(x, y)}{t^{1 / \beta_{0}}}\right)^{-\left(\alpha+\beta_{0}\right)}
$$

for any $x, y \in K$, for any $t \in\left(0, \operatorname{diam}(K)^{\beta_{0}}\right)$.

Since

$$
\frac{1}{2^{\alpha+\beta_{0}}}\left(\frac{1}{t^{\alpha / \beta_{0}}} \wedge \frac{t}{d(x, y)^{\alpha+\beta_{0}}}\right) \leq \frac{1}{t^{\alpha / \beta_{0}}}\left(1+\frac{d(x, y)}{t^{1 / \beta_{0}}}\right)^{-\left(\alpha+\beta_{0}\right)} \leq\left(\frac{1}{t^{\alpha / \beta_{0}}} \wedge \frac{t}{d(x, y)^{\alpha+\beta_{0}}}\right)
$$

for any $x, y \in K$, for any $t \in(0,+\infty)$, we may assume that

$$
C_{1}\left(\frac{1}{t^{\alpha / \beta_{0}}} \wedge \frac{t}{d(x, y)^{\alpha+\beta_{0}}}\right) \leq p_{t}(x, y) \leq C_{2}\left(\frac{1}{t^{\alpha / \beta_{0}}} \wedge \frac{t}{d(x, y)^{\alpha+\beta_{0}}}\right)
$$

for any $x, y \in K$, for any $t \in\left(0, \operatorname{diam}(K)^{\beta_{0}}\right)$.

If $\operatorname{diam}(K)=+\infty$. Since

$$
\int_{0}^{+\infty} \frac{1}{t^{1+\delta}}\left(\frac{1}{t^{\alpha / \beta_{0}}} \wedge \frac{t}{d(x, y)^{\alpha+\beta_{0}}}\right) \mathrm{d} t=\left(\frac{1}{1-\delta}+\frac{\beta_{0}}{\alpha+\delta \beta_{0}}\right) \frac{1}{d(x, y)^{\alpha+\delta \beta_{0}}},
$$

we have

$$
\begin{aligned}
& \delta\left[\frac{C_{1}}{\Gamma(2-\delta)}\left(1+\frac{(1-\delta) \beta_{0}}{\alpha+\delta \beta_{0}}\right)\right] \frac{1}{d(x, y)^{\alpha+\delta \beta_{0}}} \leq J^{(\delta)}(x, y) \\
& \leq \delta\left[\frac{C_{2}}{\Gamma(2-\delta)}\left(1+\frac{(1-\delta) \beta_{0}}{\alpha+\delta \beta_{0}}\right)\right] \frac{1}{d(x, y)^{\alpha+\delta \beta_{0}}} .
\end{aligned}
$$


If $\operatorname{diam}(K)<+\infty$. Using semi-group property, we have

$$
p_{t}(x, y) \leq \frac{C_{2}}{\operatorname{diam}(K)^{\alpha}} \text { for any } t \in\left[\operatorname{diam}(K)^{\beta_{0}},+\infty\right) \text {, for any } x, y \in K \text {. }
$$

Hence

$$
\begin{aligned}
& J^{(\delta)}(x, y)=\frac{\delta}{\Gamma(1-\delta)}\left(\int_{0}^{\operatorname{diam}(K)^{\beta_{0}}}+\int_{\operatorname{diam}(K)^{\beta_{0}}}^{+\infty}\right) \frac{1}{t^{1+\delta}} p_{t}(x, y) \mathrm{d} t \\
& \leq \frac{\delta}{\Gamma(1-\delta)}\left(\int_{0}^{\operatorname{diam}(K)^{\beta_{0}}} \frac{1}{t^{1+\delta}} C_{2}\left(\frac{1}{t^{\alpha / \beta_{0}}} \wedge \frac{t}{d(x, y)^{\alpha+\beta_{0}}}\right) \mathrm{d} t\right. \\
& \left.+\int_{\operatorname{diam}(K)^{\beta_{0}}}^{+\infty} \frac{1}{t^{1+\delta}} \frac{C_{2}}{\operatorname{diam}(K)^{\alpha}} \mathrm{d} t\right) \\
& \leq \frac{\delta}{\Gamma(1-\delta)}\left(\int_{0}^{+\infty} \frac{1}{t^{1+\delta}} C_{2}\left(\frac{1}{t^{\alpha / \beta_{0}}} \wedge \frac{t}{d(x, y)^{\alpha+\beta_{0}}}\right) \mathrm{d} t+\int_{\operatorname{diam}(K)^{\beta_{0}}}^{+\infty} \frac{1}{t^{1+\delta}} \frac{C_{2}}{\operatorname{diam}(K)^{\alpha}} \mathrm{d} t\right) \\
& =\frac{\delta}{\Gamma(1-\delta)} C_{2}\left(\left(\frac{1}{1-\delta}+\frac{\beta_{0}}{\alpha+\delta \beta_{0}}\right) \frac{1}{d(x, y)^{\alpha+\delta \beta_{0}}}+\frac{1}{\delta} \frac{1}{\operatorname{diam}(K)^{\alpha+\delta \beta_{0}}}\right) \\
& \leq \delta\left[\frac{C_{2}}{\Gamma(2-\delta)}\left(1+\frac{(1-\delta) \beta_{0}}{\alpha+\delta \beta_{0}}+\frac{1-\delta}{\delta}\right)\right] \frac{1}{d(x, y)^{\alpha+\delta \beta_{0}}} .
\end{aligned}
$$

On the other hand

$$
\begin{aligned}
& J^{(\delta)}(x, y) \geq \frac{\delta}{\Gamma(1-\delta)} \int_{0}^{\operatorname{diam}(K)^{\beta_{0}}} \frac{1}{t^{1+\delta}} p_{t}(x, y) \mathrm{d} t \\
& \geq \frac{\delta}{\Gamma(1-\delta)} \int_{0}^{\operatorname{diam}(K)^{\beta_{0}}} \frac{1}{t^{1+\delta}} C_{1}\left(\frac{1}{t^{\alpha / \beta_{0}}} \wedge \frac{t}{d(x, y)^{\alpha+\beta_{0}}}\right) \mathrm{d} t \\
& \geq \frac{\delta}{\Gamma(1-\delta)} \int_{0}^{d(x, y)^{\beta_{0}}} \frac{1}{t^{1+\delta}} C_{1}\left(\frac{1}{t^{\alpha / \beta_{0}}} \wedge \frac{t}{d(x, y)^{\alpha+\beta_{0}}}\right) \mathrm{d} t \\
& =\frac{\delta}{\Gamma(1-\delta)} \int_{0}^{d(x, y)^{\beta_{0}}} \frac{1}{t^{1+\delta}} C_{1} \frac{t}{d(x, y)^{\alpha+\beta_{0}}} \mathrm{~d} t \\
& =\delta \frac{C_{1}}{\Gamma(2-\delta)} \frac{1}{d(x, y)^{\alpha+\delta \beta_{0}}} .
\end{aligned}
$$

\section{References}

[1] Martin T. Barlow and Richard F. Bass. The construction of Brownian motion on the Sierpiński carpet. Ann. Inst. H. Poincaré Probab. Statist., 25(3):225-257, 1989.

[2] Martin T. Barlow and Richard F. Bass. On the resistance of the Sierpiński carpet. Proc. Roy. Soc. London Ser. A, 431(1882):345-360, 1990.

[3] Martin T. Barlow, Richard F. Bass, and John D. Sherwood. Resistance and spectral dimension of Sierpiński carpets. J. Phys. A, 23(6):L253-L258, 1990.

[4] Martin T. Barlow and Edwin A. Perkins. Brownian motion on the Sierpiński gasket. Probab. Theory Related Fields, 79(4):543-623, 1988.

[5] Jean Bertoin. Lévy processes, volume 121 of Cambridge Tracts in Mathematics. Cambridge University Press, Cambridge, 1996.

[6] Masatoshi Fukushima, Yoichi Oshima, and Masayoshi Takeda. Dirichlet forms and symmetric Markov processes, volume 19 of De Gruyter studies in mathematics ; 19. de Gruyter, Berlin [u.a.], 2., rev. and extended ed. edition, 2011.

[7] Alexander Grigor'yan and Takashi Kumagai. On the dichotomy in the heat kernel two sided estimates. In Analysis on graphs and its applications, volume 77 of Proc. Sympos. Pure Math., pages 199-210. Amer. Math. Soc., Providence, RI, 2008. 
[8] Alexander Grigor'yan and Meng Yang. Determination of the walk dimension of the sierpiński gasket without using diffusion. J. Fractal Geom., 5(4):419-460, 2018.

[9] Alexander Grigor'yan and Meng Yang. Local and non-local Dirichlet forms on the Sierpiński carpet. Trans. Amer. Math. Soc., 372(6):3985-4030, 2019.

[10] Jun Kigami. A harmonic calculus on the Sierpiński spaces. Japan J. Appl. Math., 6(2):259-290, 1989.

[11] Jun Kigami. Harmonic calculus on p.c.f. self-similar sets. Trans. Amer. Math. Soc., 335(2):721-755, 1993.

[12] Jun Kigami. Analysis on fractals, volume 143 of Cambridge Tracts in Mathematics. Cambridge University Press, Cambridge, 2001.

[13] Shigeo Kusuoka and Xian Yin Zhou. Dirichlet forms on fractals: Poincaré constant and resistance. Probab. Theory Related Fields, 93(2):169-196, 1992.

[14] Ivor McGillivray. Resistance in higher-dimensional Sierpiński carpets. Potential Anal., 16(3):289-303, 2002.

[15] Katarzyna Pietruska-Pałuba. Limiting behaviour of Dirichlet forms for stable processes on metric spaces. Bull. Pol. Acad. Sci. Math., 56(3-4):257-266, 2008.

[16] Ken-iti Sato. Lévy processes and infinitely divisible distributions, volume 68 of Cambridge Studies in Advanced Mathematics. Cambridge University Press, Cambridge, 1999. Translated from the 1990 Japanese original, Revised by the author.

[17] Meng Yang. Construction of Local Regular Dirichlet Form on the Sierpiński Gasket using $\Gamma$-Convergence. arXiv e-prints, page arXiv:1706.04998, Jun 2017.

[18] Meng Yang. Equivalent semi-norms of non-local Dirichlet forms on the Sierpiński gasket and applications. Potential Anal., 49(2):287-308, 2018.

Université Grenoble Alpes, CNRS UMR 5582, Institut Fourier, Gières, France.

E-mail address: yangmengqh@gmail.com 